\title{
Registro paleopalinológico de la formación San Gregorio (Barreno B4), Baja California Sur, México
}

Iris G. Galván-Escobedo, Elia Ramírez-Arriaga, Alfonso Valiente-Banuet, Ebandro Uscanga-Mortera, Edmundo García-Moya, Josué Kohashi-Shibata

Iris G. Galván-Escobedo

sirigales@gmail.com

Ebandro Uscanga-Mortera

Edmundo García-Moya

Josué Kohashi-Shibata

Posgrado en Botánica, Colegio de Postgra-

duados, Campus Montecillo, Carretera Mé-

xico-Texcoco Km. 36.5, Montecillo, Texcoco,

56230, Estado de México.

\section{Elia Ramírez-Arriaga}

Laboratorio de Palinología, Dpto. de Paleontología, Instituto de Geología, Universidad Nacional Autónoma de México, Ciudad Universitaria, 04510, Coyoacán, Ciudad de México.

\section{Alfonso Valiente-Banuet}

Departamento de Ecología de la Biodiversidad, Instituto de Ecología, Universidad Nacional Autónoma de México, Ciudad Universitaria, 04510, Coyoacán, Ciudad de México.

BOL. SOC. GEOL. MEX. 2017

VOL. 69 NO. 1

P. $35-58$

Manuscrito recibido: Noviembre 4, 2015

Manuscrito corregido: Julio 7, 2016.

Manuscrito aceptado: Julio 16, 2016.
RESUMEN

En la región de La Purísima, Comondú, Baja California Sur, se extrajo el barreno B4 de la formación San Gregorio Oligoceno superior-Mioceno inferior), el cual contiene microfósiles de origen continental y marino. El objetivo de esta investigación fue estudiar los palinomorfos recuperados del barreno y analizar su riqueza y diversidad $a$. Con base en los microfósiles continentales, se proponen los tipos de vegetación en que pudieron desarrollarse estos elementos. Así mismo, el ambiente de depósito se interpretó con base en los microfósiles marinos. La riqueza total de taxones fue de $\mathrm{n}=189$, mientras que la riqueza de los conjuntos de elementos continentales y de dinoflagelados fue $\mathrm{n}=161$ y $\mathrm{n}=28$, respectivamente. La diversidad a $\left(H^{\prime}\right)$ de elementos continentales fue de 2.61 y la uniformidad $(\mathcal{f})$ fue de 0.51. La diversidad $H^{\prime}$ del conjunto de dinoflagelados fue de 2.33 y $\mathcal{f}^{\prime}$ ' de 0.70 . La diversidad $H^{\prime}$ del conjunto total fue de 3.02 y $\mathcal{F}$ de 0.57 . El análisis de agrupamiento CONISS (constrained incremental sum of squares clustering), definió tres zonas en la columna estratigráfica del barreno. La zona A, se caracterizó por la presencia de quistes de dinoflagelados de Operculodinium y la escasez de polen-esporas. La zona B, se definió por la existencia de palinomorfos continentales; destacó la presencia de polen de Chenopodipollis, Graminidites, Liliacidites y esporas de Lusatisporis dettmannae, y los quistes de dinoflagelados Achomosphaera, Lingulodinium y Polysphaeridium. La zona C, se estableció por el registro de polen de Tubulifloridites. Los taxones de importancia cronoestratigráfica fueron Corsinipollenites, Ephedripites claricristatus, Momipites coryloides y $M$. tenuipolus. El conjunto de polen-esporas sugiere la presencia de dos palecomunidades vegetales: a nivel regional, el bosque mesófilo de montaña en las zonas altas y, a nivel local cerca de la paleocuenca de depósito, el bosque tropical caducifolio. La presencia de palinomorfos marinos en el barreno B4 sugiere que estos depósitos corresponden a un ambiente marino somero cercano al litoral.

Palabras clave: polen-esporas, dinoflagelados, diversidad, paleovegetación, formación San Gregorio.

\section{ABSTRACT}

In La Purísima region, Comondú, Baja California Sur, the borehole B4 was extracted from the San Gregorio formation (upper Oligocene-lower Miocene). It contains continental and marine palynomorphs. The aim of this study was to examine the borehole palinomorphs and analyze their richness and a diversity. We propose the vegetation types in which these elements could develop based on continental microfossils. Also the deposition environment was interpreted based on the marine microfossils. The richness for pollen-spores and dinoflagellate cysts assemblages was $n=161$ and $n=28$ respectively. The total richness was $n$ $=189$. The a-diversity $\left(H^{\prime}\right)$ of the pollen-spores assemblage was 2.61 and the evenness ( $\left.7^{\prime}\right)$ was 0.51 . The dinoflagellate cysts diversity $H^{\prime}$ was 2.33 and ' $y^{\prime}$ was 0.70 . The total diversity $H^{\prime}$ was 3.02, and 7' was 0.57. Using CONISS analysis (constrained incremental sum of squares clustering), three zones in the stratigraphic column of the borehole were defined. Zone A, was characterized by the presence of Operculodinium dinoflagellate cysts and a scarcity of pollen-spores. Zone B, was defined by continental palynomorphs, highlighting the presence of Chenopodipollis, Graminidites, Liliacidites pollen and Lusatisporis dettmannae spores, as well as Achomosphaera, Lingulodinium and Polysphaeridium dinoflagellates cysts. Zone $C$, was established by the Tubulifloridites pollen record. The chronostratigraphic pointers were: Corsinipollenites, Ephedripites claricristatus, Momipites coryloides and $M$. tenuipolus. The pollen-spore assemblages suggests the presence of two plant paleocommunities: a cloud forest in the highlands at the regional level, and a tropical deciduous forest at the local level near to the deposit basin. The presence of marine palynomorphs in the sequence suggest that these deposits correspond to a shallow marine environment near the coast.

Keywords: pollen-spores, dinoflagellates, diversity, paleovegetation, San Gregorio formation. 


\section{Introducción}

La determinación de la antigüedad y distribución de taxones a través del tiempo, es central para poder comprender los orígenes y la alta diversidad vegetal actual en México (Rzedowski, 2006). Patrones de riqueza de especies a gran escala responden en primera instancia a causales históricos (Wiens y Donoghue, 2004; Wiens y Graham, 2005; Donoghue, 2008), por lo que conocer su historia biogeográfica y la vegetación de la que formaban parte es central para entender los patrones de diversidad a escalas regionales. La transición Paleógeno/Neógeno en particular, ha sido considerada de gran importancia para la evolución de la flora y la vegetación de México (Becerra, 2005; Rzedowski, 2006; Valiente-Banuet et al., 2006).

La reconstrucción de la vegetación que existió en México durante el Paleógeno y Neógeno, ha tenido grandes avances gracias a los estudios palinoflorísticos y palinoestratigráficos llevados a cabo en paleocuencas continentales y marinas, en el norte, centro y sur del país (Langenheim et al., 1967; Graham, 1976; Biaggi, 1978; Tomasini-Ortíz y Martínez-Hernández, 1984; Quiroz-García y Martínez-Hernández, 1987; Martínez-Hernández, 1991, 1992a; Rosales-Lomelí et al., 1992; Palacios-Chávez y Rzedowski, 1993; Martínez-Hernández y Ramírez-Arriaga, 1996; Graham, 1999; Ramírez-Arriaga, 2005; Ramírez-Arriaga et al., 2006; Carrasco-Velázquez et al., 2009; Ramírez-Arriaga et al., 2012b; Ramírez-Arriaga et al., 2014a).

Existen solo cuatro localidades en el norte de México, en donde se han llevado a cabo estudios palinoestratigráficos. La primera de ellas en la Cuenca de Burgos (Eoceno medio-Eoceno superior) en los estados de Tamaulipas y Nuevo León (Martínez-Hernández et al., 1980) en donde se registró polen de Corsinipollenites, Ilexpollenites, Momipites, Nudopollis, Platycaria, entre otros. La segunda, en el estado de Sonora, incluye material de las Formaciones Tubutama (Mioceno inferior) y Baucarit (Mioceno-Plioceno) (Martínez-Hernández y Tomasini-Ortíz, 1986). En esta localidad la palinoflo- ra está compuesta por elementos afines a la flora Madro-Terciaria de clima subtropical a templado con aridez estacional que indican el inicio y evolución de comunidades adaptadas a condiciones de sequía a partir del Mioceno inferior (Martínez-Hernández, 1992b). La tercera, se encuentra en los depósitos minerales de La Perla, Chihuahua (Oligoceno superior-Mioceno), los cuales contienen polen de Alnipollenites, Chenopodipollis, Momipites, Triatripollenites, Triporopollenites, Tubilifloridites y Typha (Corona-Esquivel et al., 2010). La cuarta está en la región de La Purísima, Baja California Sur (BCS) en donde se han realizado investigaciones preliminares en la formación San Gregorio (FSG) (Martínez-Hernández y Ramírez-Arriaga, 1996, 2006; Ramírez-Arriaga et al., 2008; Ramírez-Arriaga et al., 2012a; Ramírez-Arriaga et al., 2013; Ramírez-Arriaga y Reyes-Salas, 2014; Ramírez-Arriaga et al., 2014b; Galván-Escobedo et al., 2015a; 2015b).

Los estudios de esta última localidad documentan que, aun cuando la FSG representa depósitos marinos, contiene elementos continentales tales como esporas de pteridofitas y briofitas, polen de gimnospermas y angiospermas. Por esta razón, es importante analizar con detalle la palinoflora, que podría representar a un conjunto de taxones que se desarrollaron en la península de Baja California cuando aún estaba conectada al continente. El análisis de la riqueza y diversidad de dicha palinoflora, puede ayudar a comprender los cambios en el paleoambiente que influyeron en la configuración de las paleocomunidades vegetales (diversidad $\alpha$ ).

La FSG se depositó durante el intervalo Oligoceno superior-Mioceno inferior (28.1-15.97 Ma) y se encuentra en la región de La Purísima, municipio de Comondú, BCS. En la actualidad, en esta región existe matorral xerófilo, bosque tropical caducifolio, bosque de pino encino y encinar (León de la Luz et al., 2012). La presente investigación comprende el estudio de los palinomorfos recuperados del barreno B4 de $105.40 \mathrm{~m}$ de profundidad, extraído de dicha formación. Se analizan la riqueza y diversidad a de los conjuntos paleo- 
palinológicos, dando mayor énfasis al conjunto de microfósiles continentales. Con estos datos se sugieren los tipos de vegetación que se desarrollaban durante ese intervalo geológico y se interpreta el ambiente de depósito.

\section{Marco geológico}

Durante el Cretácico, el sur de la península de Baja California se encontraba en la paleolatitud actual de Cabo Corrientes, cerca de Puerto Vallarta, Jalisco (Smith, 1991; Helenes-Escamilla y Carreño, 1999; Ledesma-Vázquez y Carreño, 2010). Esta condición se mantuvo aproximadamente del Oligoceno al Mioceno (hace 33.9 a $5.3 \mathrm{Ma}$ ) (Aranda-Gómez et al., 2000; Carreño y Helenes, 2002) hasta que, en el Mioceno medio-superior $(12-6$ Ma), el margen noroeste de México se acopló a la Placa del Pacífico y la península comenzó a migrar gradualmente hacia el norte, lejos de la Placa de Norte América (Galli-Olivier, 1993; Helenes-Escamilla y Carreño, 1999; Martín-Barajas, 2000; Helenes-Escamilla et al., 2009; Ledesma-Vázquez y Carreño, 2010). Como resultado se creó una depresión subcostal en el oeste de la costa de México, la cual es conocida como "extensión del proto-Golfo", que más tarde, durante el Holoceno (0.01 Ma), dio origen al actual Golfo de California (Galli-Olivier, 1993; Helenes-Escamilla y Carreño, 1999; Aranda-Gómez et al., 2000; Carreño y Smith, 2007; Helenes-Escamilla et al., 2009; Ledesma-Vázquez y Carreño, 2010).

Durante el Paleógeno/Neógeno gran parte del estado de BCS, estuvo cubierto por un mar somero en el cual se depositaron más de $4000 \mathrm{~m}$ de sedimentos (Alatorre, 1988). Al norte del estado, en la región de La Purísima (municipio de Comondú), la secuencia estratigráfica de estos depósitos consiste de las formaciones Tepetate (Paleoceno-Eoceno), San Gregorio (Oligoceno superior-Mioceno inferior), San Isidro (Mioceno inferior o medio), Comondú (Oligoceno superior-Mioceno medio) y La Salada (Plioceno) (Hausback, 1984; Applegate,
1986; Alatorre, 1988; Fischer et al., 1995; Carreño y Smith, 2007) (Figura 1a).

\subsection{FORMAGIÓN SAN GREGORIO}

\subsubsection{ESTRATIGRAFÍA}

La litología de la FSG ha sido descrita en la zona de La Purísima, en los arroyos Purísima, Cadajé, San Raymundo (Beal, 1948) y Cadegomo, en el rancho San Ramón y La Ventana (Heim, 1922). Esta formación está constituida por intercalaciones de lutita fosfatada, lutita silificada, diatomita, arenisca peloidal fosfatada y toba riolítica (Darton, 1921; Hausback, 1984; Kim y Barron, 1986). También incluye lutita con diatomeas, algunas de color café chocolate, que se caracterizan por su alto contenido de escamas de peces, algunos de los estratos silificados despiden un olor fétido cuando se golpean con el martillo y las capas de diatomita son blancas y polvosas (Beal, 1948).

\subsubsection{AMBIENTE DE DEPÓSITO}

Los estudios de diatomeas y crustáceos, así como los análisis de los estratos de fosforitas, sugieren que la FSG, se depositó en ambientes desde costeros hasta neríticos externos, con profundidades máximas de 200 m (Kim y Barron, 1986; Galli-Olivier, 1993; Grimm y Föllmi, 1994; Schwennicke, 1994, 1995).

\section{Métodos}

El Consejo de Recursos Minerales (actualmente Servicio Geológico Mexicano) extrajo varios barrenos de exploración minera en la zona de La $\mathrm{Pu}-$ rísima, al norte del municipio de Comondú, BCS. Uno de esos barrenos fue el B4, localizado en las coordenadas $26^{\circ} 19^{\prime} 03^{\prime \prime} \mathrm{Ny}^{1} 12^{\circ} 06^{\prime} 08^{\prime \prime} \mathrm{O}$ (Figura lb, c, d).

El barreno B4 tiene $105.40 \mathrm{~m}$ de profundidad. La litología en la base del barreno, presentó una roca ígnea en composición andesítica con sulfuro en forma diseminada asociado a microfracturas y zona de falla. El sulfuro se presenta en sus va- 


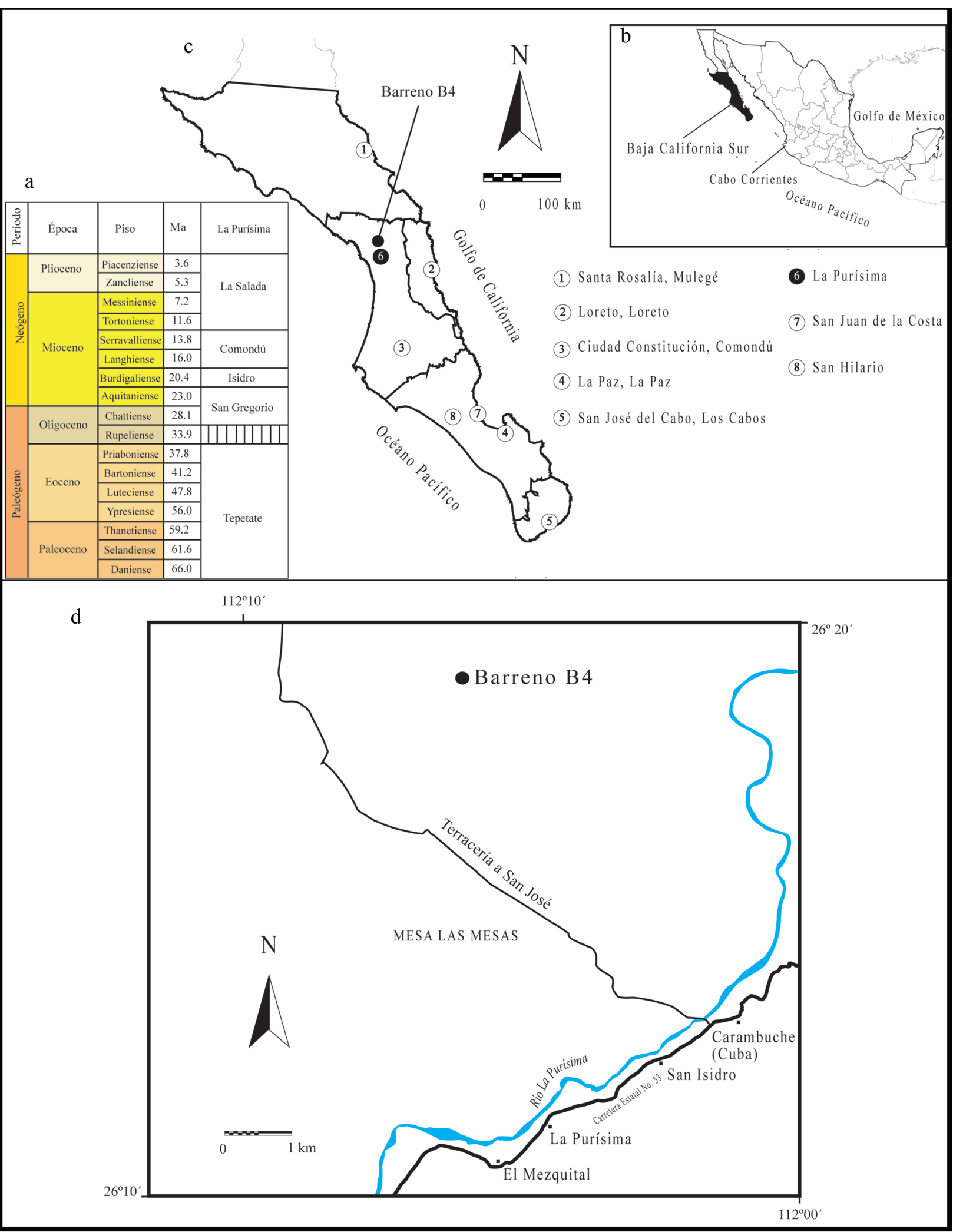

Figura 1 a) Secuencia estratigráfica del Paleógeno-Neógeno en la región de La Purísima, Baja California Sur (BCS), México (Hausback, 1984; Applegate, 1986; Alatorre, 1988; Fischer et al., 1995; Carreño y Smith, 2007); b) Localización geográfica de Baja California Sur; c) Ubicación geográfica del barreno B4; d) Detalle de la zona de estudio. 
riedades mineralógicas de pirita y calcopirita. En la parte media presenta intercalaciones de capas fosfatadas, diatomita, limolita, arenisca fosfatada, arenisca calcárea, lutita y calcilutita. La cima del barreno corresponde con terrazas aluviales con fragmentos de roca volcánica (Figura 2).

El barreno se submuestreó y se obtuvieron un total de 40 muestras (Figura 2), las cuales se procesaron por métodos estándares para la extracción de palinomorfos, con ácido clorhídrico, ácido fluorhídrico, acetólisis y flotación con politungstato de sodio. Se elaboraron laminillas permanentes con hidroxiacetil celulosa y bálsamo de Canadá, las cuales se depositaron en la colección de polen fósil del Instituto de Geología, de la Universidad Nacional Autónoma de México.

Se revisó una laminilla de cada muestra en un microscopio óptico Zeiss, con objetivo 100x y contraste de fases. En cada laminilla se contaron todos los palinomorfos continentales (polen-esporas) y marinos (dinoflagelados, acritarcas/prasinofitas, huevos de copépodos, escolecodontos y testas de microforaminíferos). Se tomaron fotomicrografías con una cámara AxioCam ICc1-Zeiss y el programa AxioVision versión 4.8.2.

Los datos se analizaron en tres conjuntos: 1) Conjunto continental (polen-esporas), 2) Conjunto de dinoflagelados y 3) Conjunto total (polen-esporas y dinoflagelados). Para contrastar los datos de diversidad entre el conjunto continental y el de dinoflagelados, se estimó la riqueza de especies, la diversidad a de especies, mediante el índice de diversidad de Shannon-Wiener $\left(H^{\prime}\right)$ con base en el logaritmo natural y su uniformidad ( $\left.\mathcal{f}^{\prime}\right)$ asociada (Pielou, 1975; Magurran, 1989). Para contrastar los valores de diversidad entre muestras, dichas estimaciones se llevaron a cabo de manera conjunta sobre el total de los elementos continentales y los dinoflagelados. Las estimaciones se realizaron con el programa Species Diversity and Richness IV (Seaby y Henderson, 2007).

Con los datos de las riquezas de palinomorfos continentales y marinos se estimó el Índice de Palinomorfos Marinos (IPM) modificado de Helenes-Escamilla et al. (1998). El IPM asociado a la presencia de los taxones de dinoflagelados, se usó para interpretar el tipo de ambiente de depósito del barreno B4. El IPM es la razón entre la riqueza de palinomorfos marinos sobre la de palinomorfos continentales y su expresión es la siguiente (Carrillo-Berumen et al., 2013):

$$
I P M=(R m /(R m+R c)) * 100
$$

En donde $R m$ es la riqueza de palinomorfos marinos considerados como el número de taxones por muestra y $R c$ es la riqueza de palinomorfos continentales. Los valores altos del IPM indican un ambiente marino, los valores bajos indican la influencia de aguas salobres, ambientes paludales costeros, estuarinos o deltaicos y los valores nulos representan ambientes exclusivamente continentales (Carrillo-Berumen et al., 2013).

Con la finalidad de observar la distribución de los taxones registrados en cada una de las muestras, se elaboraron diagramas polínicos con las frecuencias absolutas; los porcentajes se graficaron solo en aquellas muestras cuyo total fue mayor de 45, en el caso del conjunto continental, y mayor de 20 en los dinoflagelados. Se definieron palinozonas en la columna estratigráfica del barreno B4 con un análisis de agrupamiento CONISS (constrained incremental sum of squares clustering) (Grimm, 1987), para el cual se tomaron en cuenta solo los datos de elementos continentales y de dinoflagelados y se excluyeron los grupos sobrerrepresentados como fueron las acritarcas/prasinofitas, huevos de copépodos, escolecodontos y testas de microforaminíferos. Los diagramas polínicos y el CONISS se realizaron en el programa Tilia versión 1.7.16 (Grimm, 2011).

\section{Resultados}

De las 40 muestras analizadas, 30 muestras presentaron palinomorfos con un buen grado de conservación (Figura 2; Tabla 1). Se contaron un total de 25470 palinomorfos. El total por muestra fue variable: 10 tuvieron más de 1000 palinomor- 
fos (Pb-10397, Pb-10372, Pb-10374, Pb-10375, Pb-10379, Pb-10381, Pb-10382, Pb-10383, Pb10384, Pb-10386), seis entre 571 y 983 (Pb-10366,
Pb-10368, Pb-10371, Pb-10377, Pb-10380, Pb10390), 11 muestras entre 138 a 488 (Pb-10358, Pb-10369, Pb-10370, Pb-10373, Pb-10376,

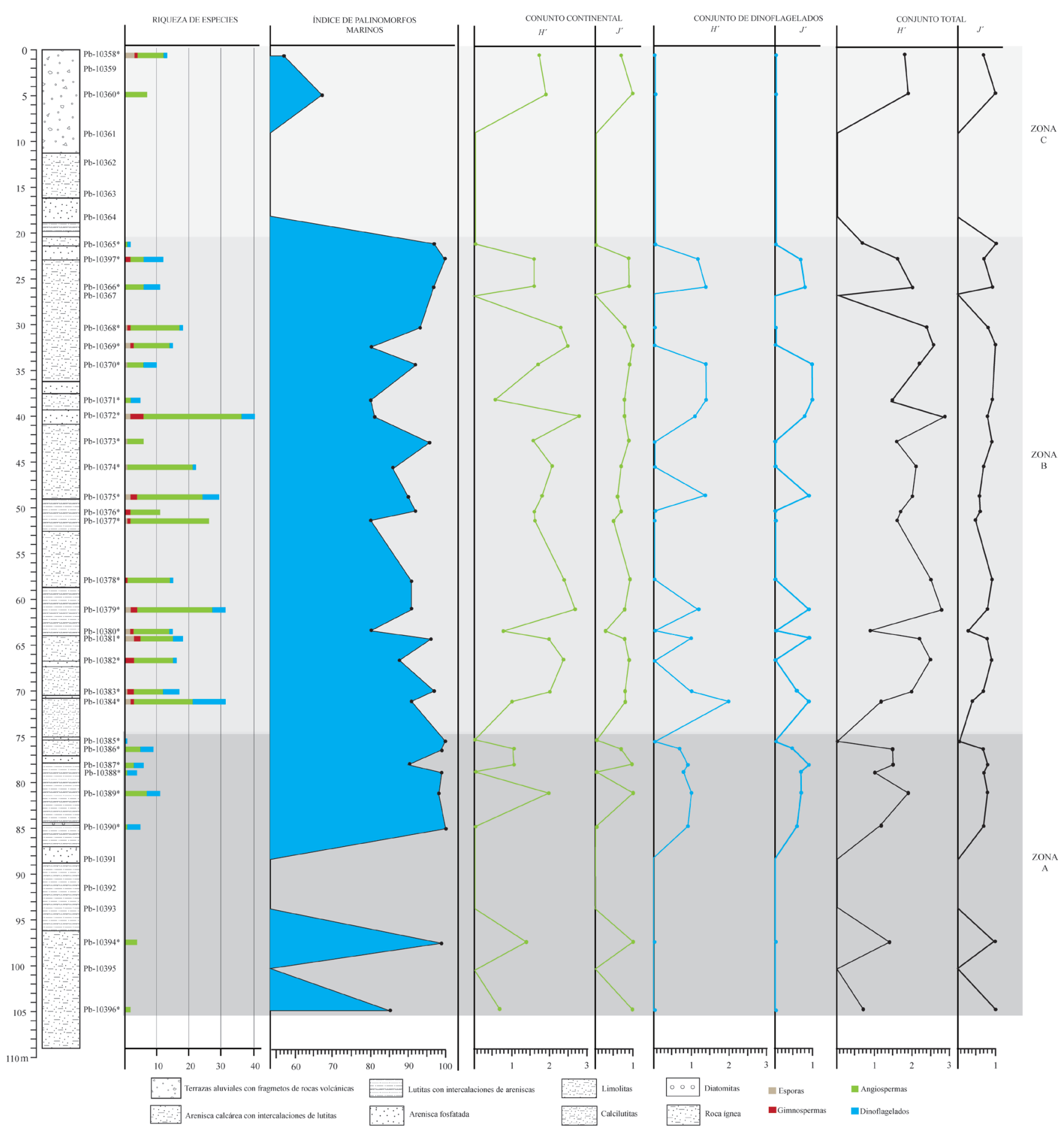

Figura 2 Litología del barreno B4 de la formación San Gregorio, La Purísima, Baja California Sur (BCS), asociado a la riqueza de especies, índice de palinomorfos marinos, índice de diversidad de especies $\alpha\left(H^{\prime}\right)$ y uniformidad $\left(J^{\prime}\right)$. Los números $\mathrm{Pb}$, indican el número de muestra y la profundidad de cada una. $(*)=$ presencia de palinomorfos. 
Tabla 1. Palinomorfos continentales y marinos registrados el barreno B4 de la formación San Gregorio, La Purísima, Comondú, Baja California Sur. Los números entre paréntesis indican el total de especies que se registraron en cada taxón. Los números entre corchetes indican los valores totales de la riqueza de especies, el índice de diversidad de especies $\alpha\left(H^{\prime}\right)$ y la uniformidad $\left(J^{\prime}\right)$ en cada uno de los conjuntos paleopalinológicos.

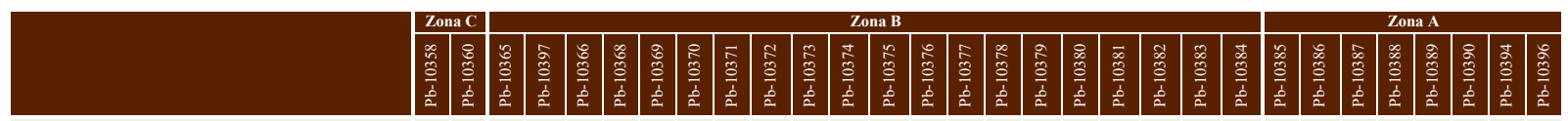

Esporas Triletes

cf. Camarazonosporites Pant ex Potonié 1956 emend. Klaus

1960 (1)

Cicatricosisporites Potonié \& Gelletich 1933 (1)

Deltoidospora Miner 1935 (1)

Granulatisporites Ibrahim 1933 emend. Potonié and Kremp

1954 (1)

Leptolepidites Couper 1953 emend. Norris 1968 (1)

Lusatisporis dettmannae (Drug) Srivastava 1972 (1)

Lycopodiacidites Couper, 1953 emend. Potonié 1956 (1)

Stereisporites Pflug 1953 (2)

Undulatisporites Pflug 1953 (1)

Esporas Monoletes

Polypodiisporites Potonié 1931 in Potonié \& Gelletich 1933

ex Potonié 1956 (1)

Gimnosperma
Bisacados

Pinuspollenites Raatz 1938 ex Potonié 1958 (1)

Poliplicas

Ephedripites Bolkhovitina 1953 (3)

Ephedripites (Distachyapites) claricristatus (Shakmunde)

Krutzsch 1970 (1)

Ephedripites aff. krempii Kedves 1977 (1)

Angiospermas

Monocotiledóneas

Monosulcado

Liliacidites Couper 1953 (9)

Longapertites Van Hoeken-Klinkenberg 1964 (1)

Tipo 1 foveolado (1)

Tipo 2 foveolado rugulado (1)

Tipo 3 psilado con patrón microrreticulado (1)

Monoporados

Graminidites Cookson 1947 ex Potonié 1960 (1)

Dicotiledóneas

Tricolpados

silatricolpites Van der Hammen ex Van der Hammen \&Wymstra 1964 (3)

Quercoidites Potonié, Thomson \& Pflug ex Potonie

1960 (3)

Retitricolpites (Van der Hammen) Pierce 1961 (9)

Tipo 1 foveolado suprarrugulado (1)

Tipo 2 microfoveolado (1)

Brevicolpados

Fraxinoipollenites Potonié 1960 (1)

Tipo 1 suprarrugulado (1)

Estefanocolpado

Tipo cf. Labiatae (1)

Tipo 1 psilado perforado (1)

Heterocolpados

Tipo Melastomataceae (1)

Tricolporoidados

Tipo 1 reticulado heterobrocado (1)

Tipo 2 supramicroverrugado (1)

Tipo 3 supramicroverrugado a rugulado (1)

ipo 4 suprarugulado (1)

Tricolporoidados-Tricolporados

Leguminosaepites Baks \& Deb 1976 (10)

Quercoidites Potonié, Thomson \& Pflug ex Potonie 1960 (4)

Tricolporados

Foveotricolporites Pierce 1961 (7)

Margocolporites Ramanujam 1966 ex Srivastava 1969b Margocolporties \& Mildenhall 1984 (2)

Myrtaceidites Cookson \& Pike 1954 ex Potonié 1960 (3) Psilatricolporites Van der Hammen 1956 ex Van der

Hammen \& Wes Va 1964 (4)

Retitricolporites Puri 1963 (11)

cf. Rugotricolporites Gonzalez (2)

Striatricolporites Van der Hammen 1956 ex Leidelmeyer

$1966(5)$

Tricolporopollenites Pflug \& Thomson in Thomson \&

Pflug 1953 (4)

Tricolporopollenites cf. retimulatus (1)

Tubulifloridites Cookson 1947 ex Potonié 1960 (4)

Tipo Anacardiaceae (3)

Tipo Euphorbiaceae (1)

Tipo Polygonaceae (Fagopyrum) (1)

Tipo Rosaceae (1)

Tipo 1 reticulado heterobrocado (1)

Tipo 2 microrreticulado (1)

Tipo 3 microrreticulado a microfoveolado (1)

Tipo 4 suprarugulado (1) 
Tabla 1. Continuación.

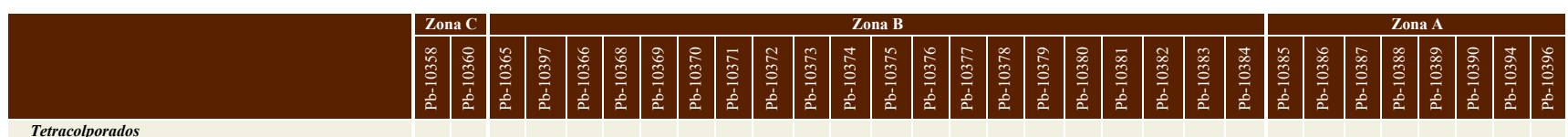

Tetracolporados

Tipo Anacardiaceae (Rhus sp.) (1)

Brevicolporados

Rugulitriporites Muller 1968 (1)

Tipo Sterculiaceae (1)

Tipo Tiliaceae (1)

Tripo 1 reticulado heterobrocado (1)

Perisincolporados

Malpighiaceoidites Takahashi \& Jux 1989 (1)

Diporados

Tipo Moraceae (1)

Triporados

Caryapollenites Raatz (1937) 1938 ex Potonié 1960 (1)

Corsinipollenites Nakoman 1965 (5)

Corsinipollis sp. (1)

Momipites Wodehouse 1933 (2)

Momipites coryloides Wodehouse 1933 (1)

Momipites microcoryphaeus (Potonié) Nichols 1973 (1)

Momipites tenuipolus Anderson 1960 (1)

Tipo Betulaceae (1)

Tipo Celtis (1)

Tipo Moraceae (1)

Tipo 1 psilado (2)

Tipo 2 supraverrugado-rugulado (1)

Tetraporados

Tipo Betulaceae (Alnus) (1)

Tipo Malpighiaceae (2)

Tetra-Penta-Hexaporados

Ulmipollenites Wolf 1934 (5)

Periporados

Chenopodipollis Krutzsch 1966 (1)

Tipo Polemoniaceae (1)

Tipo 1 foveolado (1)

Tipo 2 suprarugulado (1)

Fungosporas

Dinoflagelados

Orden Gonyaulacales

Achomosphaera Evitt 1963 (1)

cf. Cleistosphaeridium Davey, Downie, Sarjeant \& Williams

Cordosphaeridium (Eisenack 1963) (1)

Hystrichokolpoma rigaudae Deflandre \& Cookson 1955 (1) Lingulodinium Wall 1967 (1)

Operculodinium Wall 1967 (1)

cf. Pilosidinium Courtinat 1989 (1)

Polysphaeridium Davey \& Williams 1966 (1)

Spiniferites (Hafniasphaera) Hansen 1977 (1)

Spiniferites mirabilis (Rossignol) Sarjeant (1)

Spiniferites pseudofurcatus (Klumpp 1953) (1)

Spiniferites ramosus (Ehrenberg 1838) (1)

Tuberculodinium aff. vancampoae (Rossignol 1962) (1)

Otros dinoflagelados

Orden Peridiniales

Palaeocystodinium Alberti, 1961 (1)

Lejeunecysta Artzner \& Dörhöfer 1978 (6)

Lejeunecysta cinctoria Bujak in Bujak et al. 1980 (1)

Selenopemphix Benedek 1972 (1)

Otros dinoflagelados (5)

Otros elementos marino

Acritarcas y Prasinofitas

Huevos de copépodo

Escolecodontos y testas de microforaminíferos TOTAL

Índice de Palinomorfos Marinos

Conjunto

Riqueza [189]

continental y de Diversidad de especies $\alpha\left(H^{\prime}\right)[3]$

dinoflagelados Uniformidad $\left(J^{\prime}\right)[0.6]$ Riqueza [161]

$\begin{array}{ll}\text { Conjunto Diversidad de especies } \alpha\left(H^{\prime}\right) & \text { [2.6] }\end{array}$ Uniformidad $\left(J^{\prime}\right)[0.5]$

Conjunto de

Riqueza [28]

dinoflagelados Diversidad de especies $\alpha\left(H^{\prime}\right)[2.3]$ Uniformidad $\left(J^{\prime}\right)[0.7]$

\begin{tabular}{ll|llllllllllllllllllllllllllll}
75 & 25 & 33 & 1749 & 504 & 834 & 202 & 254 & 467 & 868 & 341 & 992 & 1008 & 378 & 486 & 288 & 1210 & 627 & 1772 & 874 & 956 & 2353 & 251 & 1327 & 317 & 159 & 367 & 646 & 372 & 56
\end{tabular}

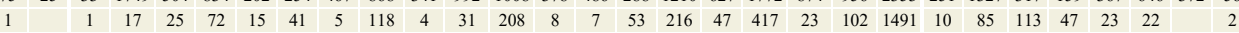

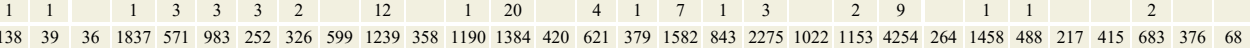

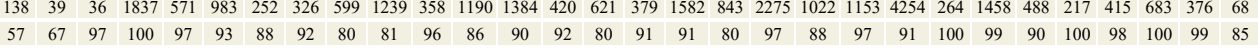

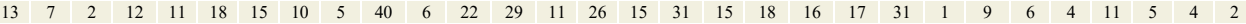
$\begin{array}{lllllllllllllllllllllllllllllll}1.8 & 1.9 & 0.7 & 1.6 & 2.1 & 2.4 & 2.6 & 2.2 & 1.5 & 2.9 & 1.6 & 2.1 & 2.1 & 1.6 & 1.6 & 2.5 & 2.8 & 0.9 & 2.2 & 2.5 & 2 & 1.3 & 0 & 1.5 & 1.5 & 1 & 1.9 & 1.2 & 1.4 & 0.7\end{array}$ $\begin{array}{lllllllllllllllllllllllllllllllllllll}0.7 & 1 & 1 & 0.7 & 0.9 & 0.8 & 1 & 0.9 & 0.9 & 0.8 & 0.9 & 0.7 & 0.6 & 0.7 & 0.5 & 0.9 & 0.8 & 0.3 & 0.8 & 0.9 & 0.7 & 0.4 & 0 & 0.7 & 0.8 & 0.7 & 0.8 & 0.7 & 1 & 1\end{array}$ \begin{tabular}{|l|l|l|l|l|l|l|l|l|l|l|l|l|l|l|l|l|l|l|l|l|l|l|l|l|l|l|l|l|l|l|}
12 & 7 & 1 & 6 & 6 & 17 & 14 & 6 & 2 & 36 & 6 & 21 & 24 & 11 & 26 & 14 & 27 & 14 & 15 & 15 & 12 & 21 & 0 & 5 & 3 & 1 & 7 & 1 & 4 & 2 \\
\hline
\end{tabular}

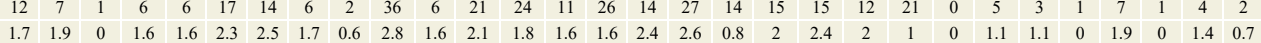
$\begin{array}{llllllllllllllllllllllllllllllllllllll}0.7 & 1 & 0 & 0.9 & 0.9 & 0.8 & 1 & 1 & 0.8 & 0.8 & 0.9 & 0.7 & 0.6 & 0.7 & 0.5 & 0.9 & 0.8 & 0.3 & 0.8 & 0.9 & 0.8 & 0.3 & 0 & 0.7 & 1 & 0 & 1 & 0 & 1 & 1\end{array}$ $\begin{array}{llllllllllllllllllllllllllllllllll}1 & 0 & 1 & 6 & 5 & 1 & 1 & 4 & 3 & 4 & 0 & 1 & 5 & 0 & 0 & 1 & 4 & 1 & 3 & 1 & 5 & 10 & 1 & 4 & 3 & 3 & 4 & 4 & 0 & 0\end{array}$ \begin{tabular}{lllllllllllll|l|l|l|l|l|l|l|l|l|l|l|l|l|l|l|l|l|l}
0 & 0 & 0 & 1.2 & 1.4 & 0 & 0 & 1.4 & 1 & 1.1 & 0 & 0 & 1.4 & 0 & 0 & 0 & 1.2 & 0 & 1 & 0 & 1 & 2.1 & 0 & 0.7 & 0.9 & 0.8 & 1 & 0.9 & 0 & 0
\end{tabular} 
Pb-10378, Pb-10385, Pb-10387, Pb-10388, Pb10389, Pb-10394), y tres menos de 100 (Pb-10360, $\mathrm{Pb}-10365, \mathrm{~Pb}-10396)$.

$\mathrm{Al}$ considerar el número total de palinomorfos contados, dominaron los microfósiles marinos en $92 \%$ los cuales incluyen dinoflagelados, acritarcas/ prasinofitas, huevos de copépodo, escolecodontos y testas de microforaminíferos. Los elementos continentales, tales como polen y esporas, se presentaron en menor cantidad y constituyeron el $6 \%$ del total. Las fungosporas representaron el $2 \%$ (Figura 3).

\subsection{DIVERSIDAD Y RIQUEZA}

\subsubsection{CONJUNTO PALEOPALINOLÓGICO CONTINENTAL}

El conjunto continental estuvo conformado por polen y esporas de pteridofitas, los cuales se encontraron en mónadas. No se registraron tétradas o políadas.
La riqueza de especies del conjunto continental fue de 161 taxones. La mayor riqueza de formas continentales por muestra fue de 36 taxones $(\mathrm{Pb}$ 10372) y la menor de 1 (Pb-10365, Pb-10385 y $\mathrm{Pb}-10390)$. La diversidad a de especies $\left(H^{\prime}\right)$ para todas las muestras que presentaron taxones continentales fue de 2.61 y por muestra osciló entre 0 (Pb-10365, Pb-10385 y Pb-10390) y 2.76 (Pb10372). La uniformidad ( $\left.\mathcal{f}^{\prime}\right)$ fue de 0.51 en total, con valores por muestra entre $0(\mathrm{~Pb}-10365, \mathrm{~Pb}-$ 10385, Pb-10388 y Pb-10390) y 1 (Pb-10387, Pb10394 y Pb-10396) (Figura 2; Tabla 1).

Las angiospermas representaron el $93 \%$ del conjunto continental, de éstas el $82 \%$ fueron dicotiledóneas y el 18 \% monocotiledóneas. Las angiospermas que sobresalieron por sus altas frecuencias fueron: Chenopodipollis, Graminidites, Liliacidites y Tubulifloridites. Otras angiospermas registradas en un menor número de muestras fueron Foveotricolporites, tipo Polygonaceae (Fagopyrum), Psi-

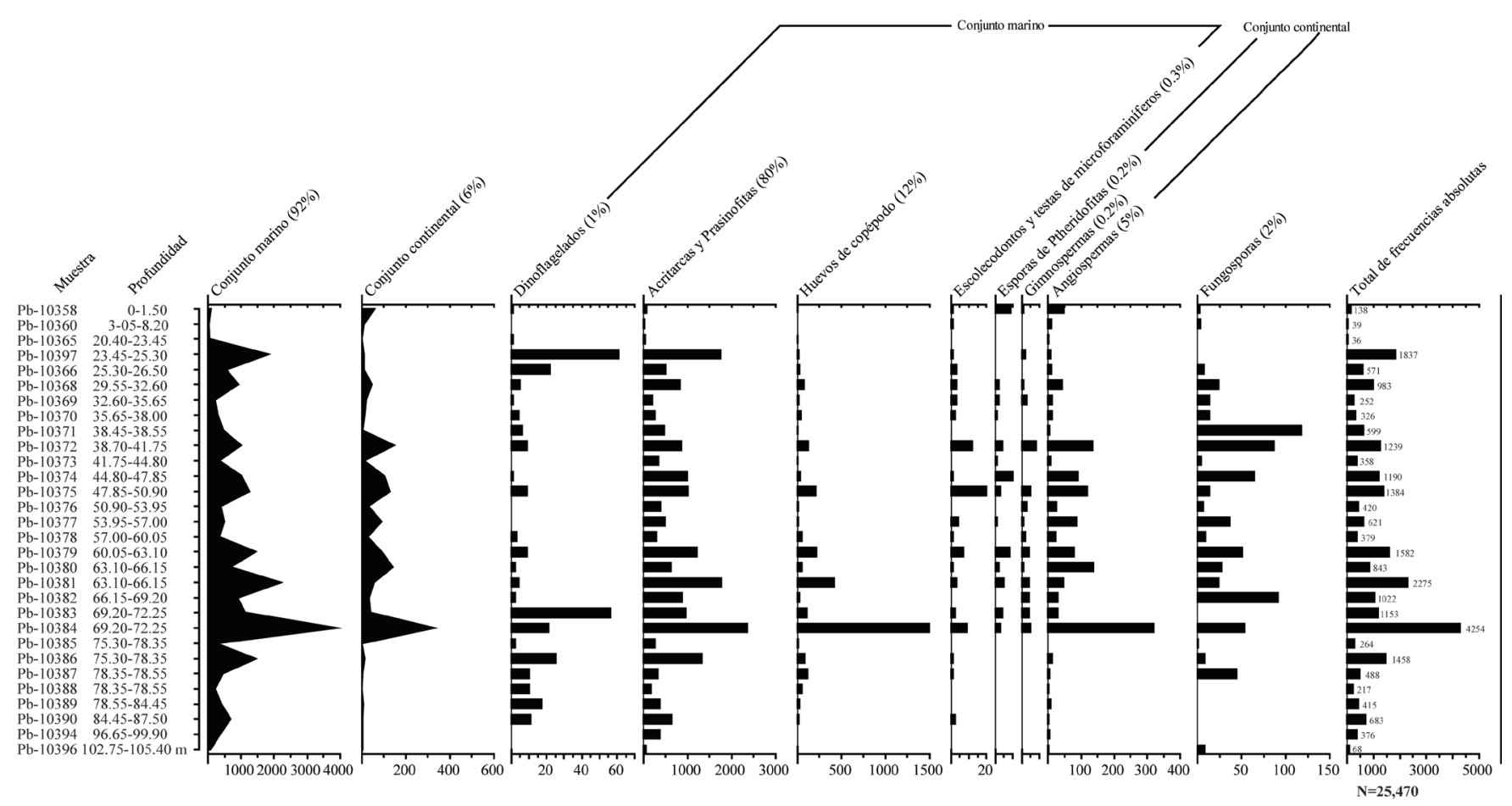

Figura 3 Resumen del conjunto paleopalinológico total del barreno B4, formación San Gregorio, La Purísima, Baja California Sur (BCS). Los números entre paréntesis indican el porcentaje que ocupa cada grupo con respecto al total de palinomorfos contados. 
latricolpites, Psilatricolporites, Quercoidites, Retitricolporites, tipo Sterculiaceae y Ulmipollenites (Figuras 4, 5a y 6$)$.

Las gimnospermas ocuparon el $3 \%$ del conjunto continental y estuvieron representadas por los taxones Ephedripites, Ephedripites claricristatus, Ephedripites aff. krempii y Pinuspollenites (Figuras 4, 5a y 6).

Las esporas de pteridofitas y briofitas representaron el $4 \%$ del conjunto. Entre éstas destacó Lusatisporis dettmannae (Figuras 4, 5a y 6). La Tabla 2, muestra los principales caracteres morfológicos de los granos de polen que presentaron mayor frecuencia en los grupos de esporas, angiospermas y gimnospermas, dentro del conjunto paleopalinológico continental.

Aunque se registraron en un número pequeño de muestras, se encontraron cuatro taxones de importancia cronoestratigráfica, uno a nivel de género: Corsinipollenites; y tres a nivel de especie: Ephedripites claricristatus, Momipites coryloides y Momipites tenuipolus (Figuras 4, 5a y 6).

\subsubsection{CONJUNTO PALEOPALINOLÓGICO DE DINOFLAGELADOS}

El conjunto de palinomorfos marinos, en general, estuvo dominado por acritarcas/prasinofitas y huevos de copépodo. La presencia de dinoflagelados de los órdenes Gonyaulacales y Peridiniales (Figuras 4, 5b y 7), representó el $1 \%$ del conjunto (Figura 3; Tabla 1).

La riqueza de especies de los dinoflagelados fue de 28 taxones. El mayor número de taxones por muestra fue de $10(\mathrm{~Pb}-10384)$ y el menor de 1 (Pb-10358， Pb-10365， Pb-10368， Pb-10369, Pb-10374, Pb-10378, Pb-10380, Pb-10382, Pb10385). La diversidad a de especies $\left(H^{\prime}\right)$ estimada para el total del conjunto de quistes fue de 2.33, cuyos valores por muestra oscilaron entre 0 (Pb-10358, Pb-10360, Pb-10365, Pb-10368, Pb-10369, Pb-10373, Pb-10374, Pb-10376, Pb10377, Pb-10378, Pb-10380, Pb-10382, Pb10385, Pb-10394, Pb-10396) y 2.06 (Pb-10384); mientras que la uniformidad ( $\left.\mathcal{f}^{\prime}\right)$ para todo el barreno fue de 0.70 y varió entre 0 y 1 ( $\mathrm{Pb}-10370)$ (Figura 2; Tabla 1).

Los taxones que dominaron el conjunto total de dinoflagelados fueron: Achomosphaera, Lingulodinium, Operculodinium y Polysphaeridium (Figuras 4, 5b y 7).

\subsubsection{CONJUNTO PALEOPALINOLÓGICO CONTINENTAL Y DE DINOFLAGELADOS}

$\mathrm{Al}$ considerar los datos del conjunto continental y de dinoflagelados, la riqueza total de especies en el barreno fue de 189 taxones. El mayor número de taxones por muestra fue de $40(\mathrm{~Pb}-10372)$ y la menor fue de 1 (Pb-10385). La diversidad a de especies $\left(H^{\prime}\right)$ de todo el barreno fue de 3.02 con una variación entre muestras desde $0(\mathrm{~Pb}-10385)$ y hasta 2.89 (Pb-10372), mientras que la uniformidad $\left(\mathcal{F}^{\prime}\right)$ fue de 0.57 en total y por muestra osciló entre 0 (Pb-10385) y 1 (Pb-10365, Pb-10394 y Pb10396) (Figura 2; Tabla 1).

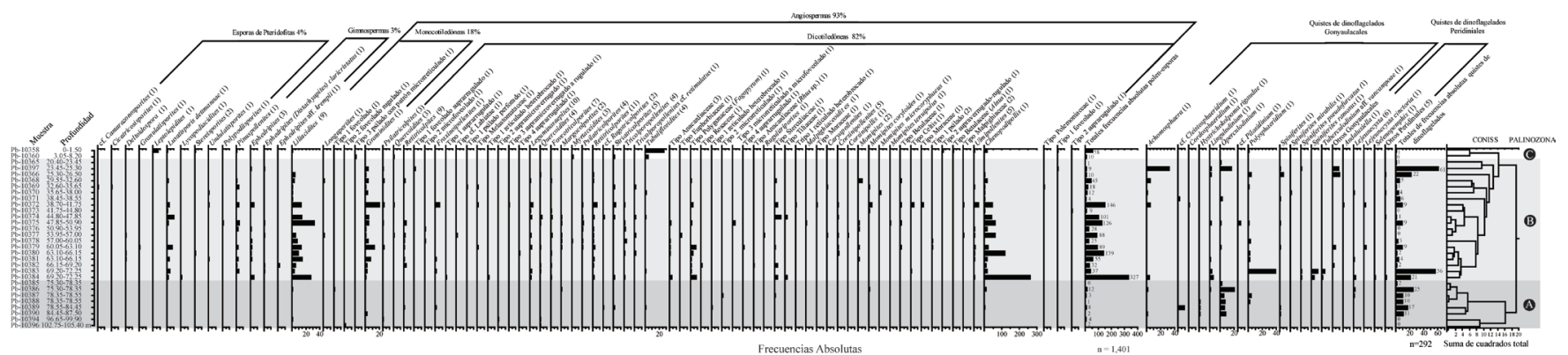

Figura 4 Palinozonas definidas en la columna estratigráfica del barreno B4 de la formación San Gregorio, La Purísima, Baja California Sur, de acuerdo al análisis CONISS efectuado con base en las frecuencias absolutas de los conjuntos continental y de dinoflagelados. Los números entre paréntesis indican el total de especies que se agrupa en cada taxón. 


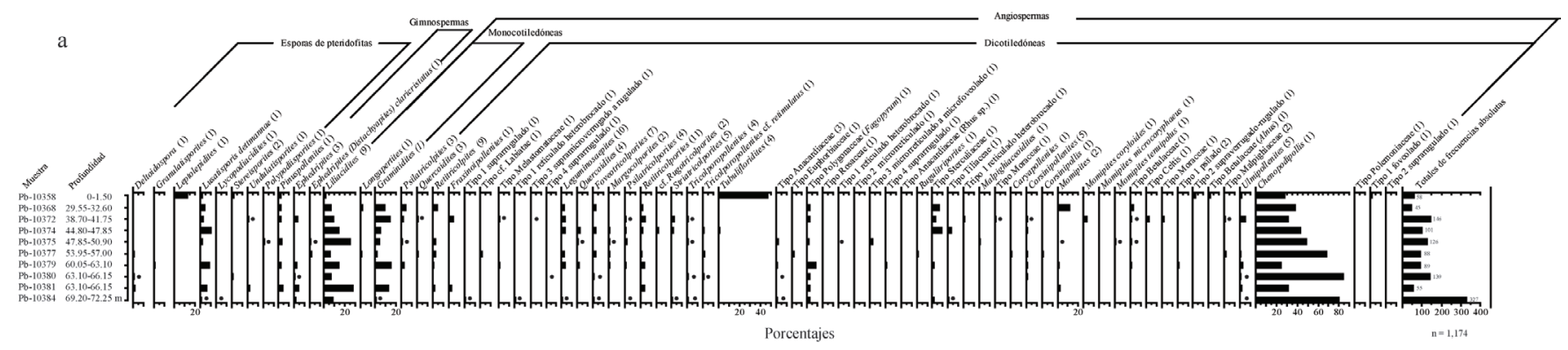

b

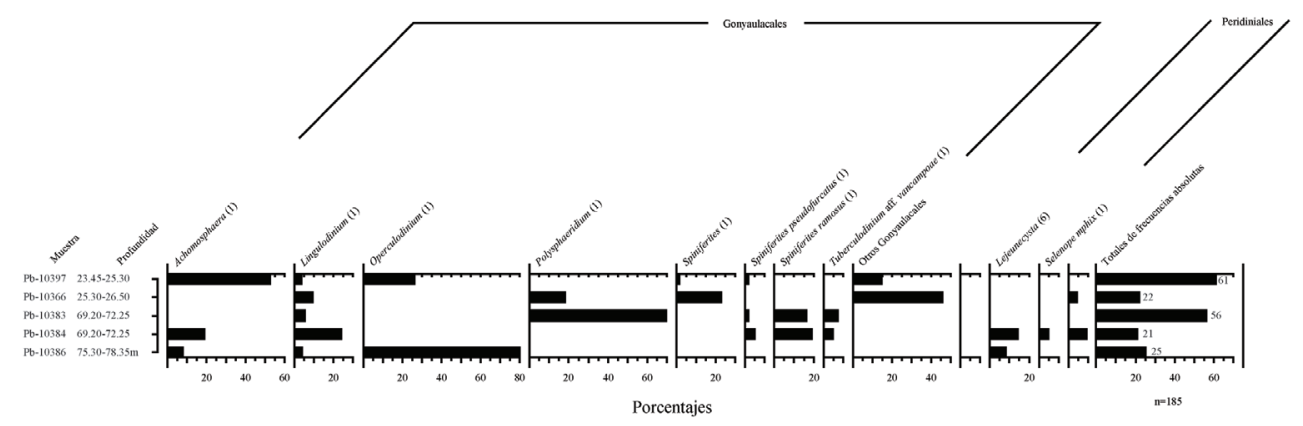

Figura 5 a) Porcentajes de los taxones continentales con frecuencias absolutas mayores a 45; b) porcentajes de los taxones de dinoflagelados con frecuencias absolutas mayores a 20. Las barras señaladas con un punto negro corresponden a porcentajes menores a 1. Los números entre paréntesis indican el total de especies que se encontraron en cada taxón.

\subsection{PALINOZONAS DE LA GOLUMNA ESTRATIGRÁFICA DEL BARRENO B4}

El análisis CONISS definió tres palinozonas a lo largo de la columna estratigráfica del barreno B4 (Figura 4):

1) La zona A (Pb-10396 a Pb-10385), se caracterizó por un predominio de dinoflagelados de Operculodinium, y bajas frecuencias de cf. Cleistosphaeridium e Hystrichokolpoma rigaudae, y por la presencia restringida de elementos continentales, entre los que se encontraron Chenopodipollis, Graminidites, Fraxinoipollenites, Liliacidites, Margocolporites, Momipites coryloides, Quercoidites, Retitricolpites, cf. Rugutricolporites, tipo Sterculiaceae, Striatricolporites y Tubulifloridites.

2) La zona B (Pb-10384 a 10365) se definió por la presencia de los taxones continentales dominantes: Chenopodipollis, Graminidites, Liliacidites y Lusatisporis dettmannae y los dinoflagelados Achomosphaera, Lingulodinium y Polysphaeridium.
Además de estos taxones, en la Figura 4 se puede observar que en esta zona se registraron la mayoría de los elementos del conjunto continental y de dinoflagelados.

3) La zona C (Pb-10360-Pb-10358) sobresalió Tubulifloridites, y hay baja frecuencia de polen de Chenopodipollis, esporas de Leptolepidites y dinoflagelados del género Polysphaeridium (Figura $4)$.

\section{3. ÍNDICE DE PALINOMORFOS MARINOS (IPM)}

La zona A del barreno presentó los mayores IPM; sobresalen las muestras $\mathrm{Pb}-10394, \mathrm{~Pb}-10390$ y $\mathrm{Pb}-$ 10385, con valores de 100 en cada una de ellas. En la zona B, se observó un comportamiento cíclico del IPM con valores que oscilaron entre 80 y 99, el máximo valor fue en la muestra $\mathrm{Pb}-10397$ y el mínimo en las muestras Pb-10380, Pb-10377 y $\mathrm{Pb}-10371$. La zona $\mathrm{G}$ se caracterizó por que presentó los valores del IPM más bajos, en las muestras $\mathrm{Pb}-10358$ que obtuvo un valor de 56, y en la $\mathrm{Pb}-10360$ con un índice de 67. 

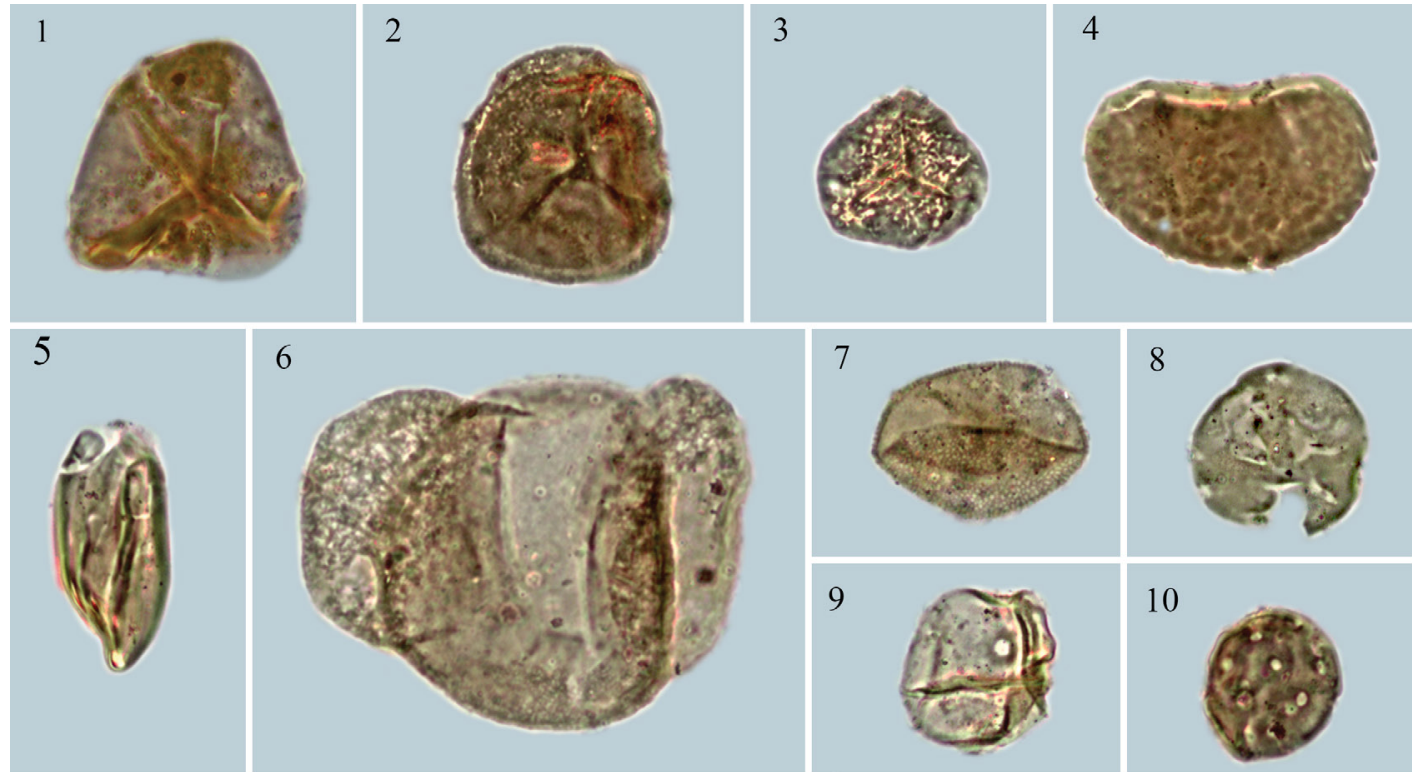

8

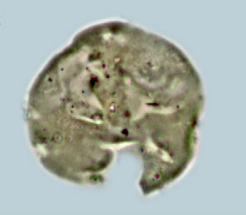

9

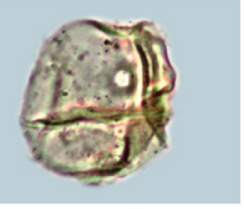

10
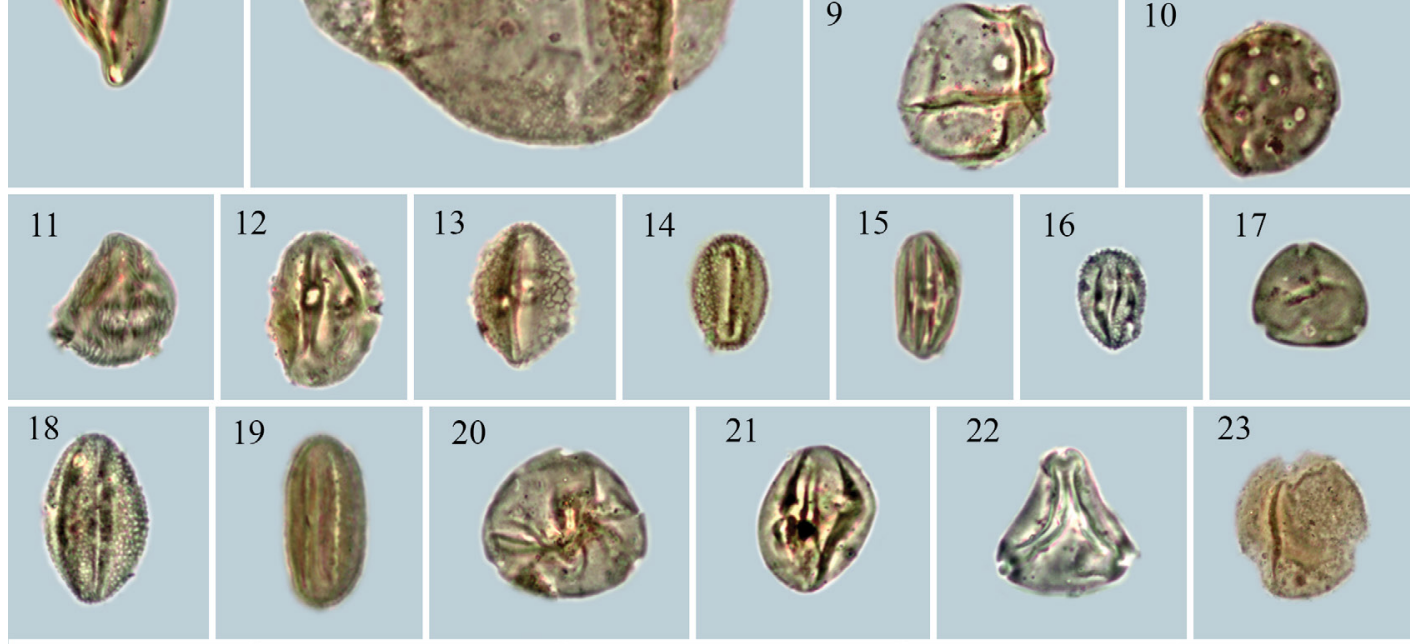

16
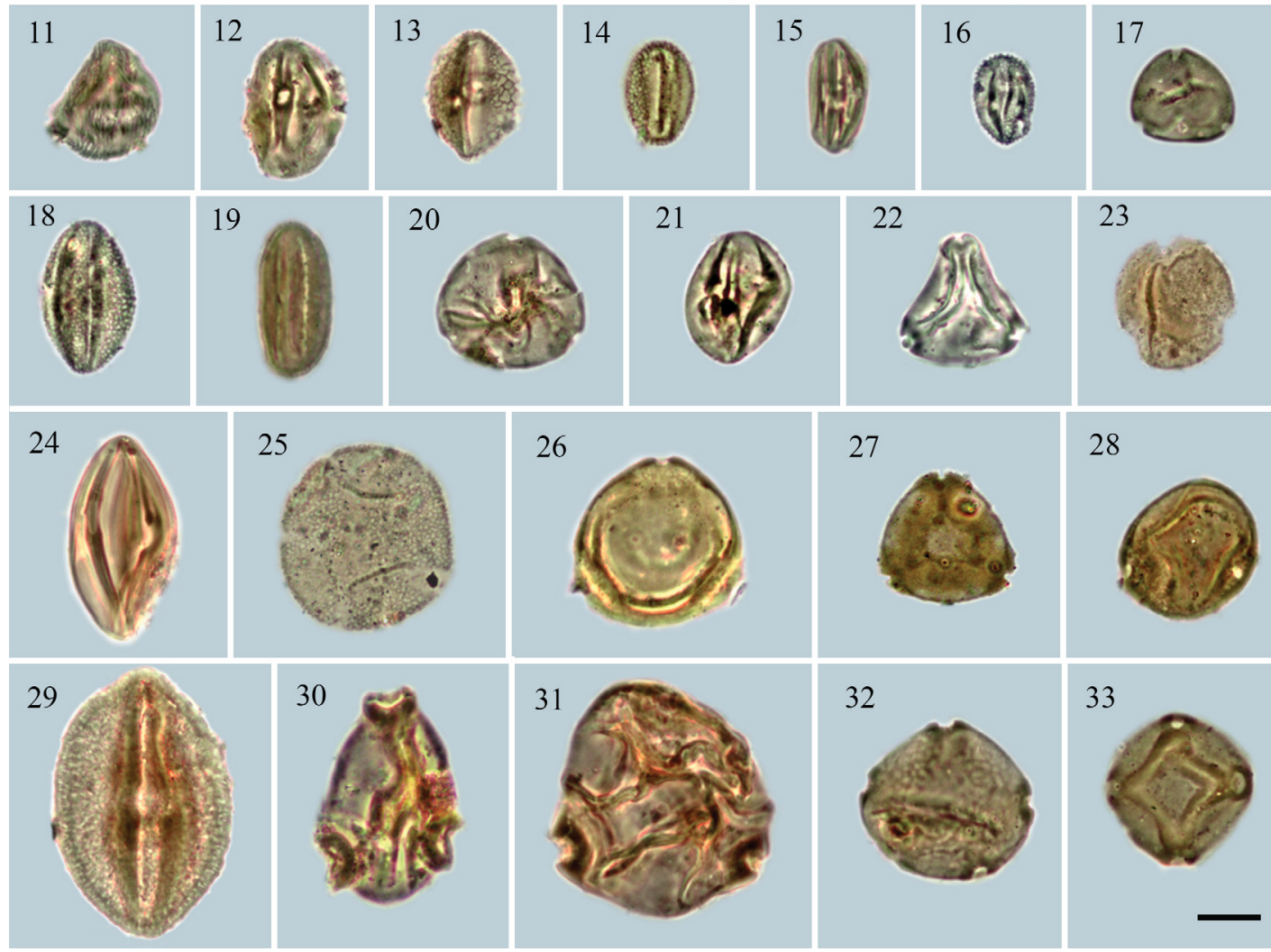

23
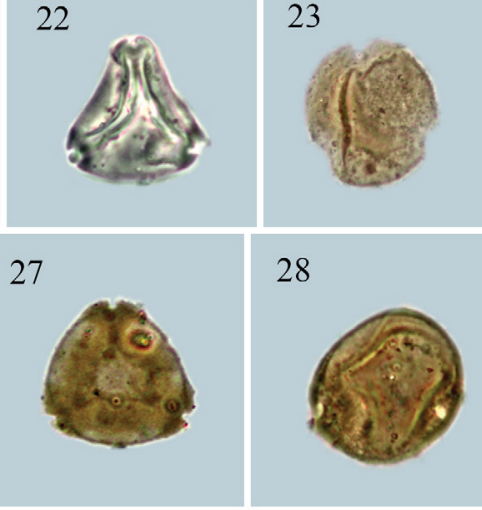

Figura 6 Palinomorfos continentales del barreno B4, formación San Gregorio, La Purísima, Baja California Sur. 1) Deltoidospora sp., $\mathrm{Pb}-10377$; 2) Lusatisporis dettmannae, $\mathrm{Pb}-10368$; 3) Lycopodiacidites sp., $\mathrm{Pb}-10384$; 4) Polypodiisporites sp., $\mathrm{Pb}-10375$; 5) Ephedripites claricristatus, $\mathrm{Pb}-10382$; 6) Pinuspollenites sp., Pb-10372; 7) Liliacidites sp. 3, Pb-10366; 8) Momipites tenuipolus, $\mathrm{Pb}-10375$; 9) Graminidites sp., Pb-10366; 10) Chenopodipollis sp., Pb-10372; 11$)$ Striatricolporites sp. 5, Pb-10384; 12) Rugulitriporites sp., Pb-10377; 13) Retitricolporites sp. 6, Pb-10372; 14) Retitricolpites sp. 2, Pb-10382; 15) Tipo Melastomataceae, $\mathrm{Pb}-10369$; 16) Retitricolporites sp. 5, Pb-10379; 17) Momipites coryloides, $\mathrm{Pb}-10369$; 18) Retitricolpites sp. 1, Pb-10368; 19) Estefanocolpado perforado, $\mathrm{Pb}-10369 ; 20$ ) Momipites microcoryphaeus, $\mathrm{Pb}-10379 ; 21)$ Psilatricolporites sp. 3, Pb-10376; 22) Myrtaceidites sp. 3, Pb-10378; 23) Quercoidites sp. 1, $\mathrm{Pb}-10366$; 24) Psilatricolporites sp. 4, Pb-10372; 25) Fraxinoipollenites sp., Pb-10372; 26) Tipo Betulaceae, $\mathrm{Pb}-10372 ; 27)$ Myrtaceidites sp. 1, Pb-10360; 28) Caryapollenites sp., Pb-10369; 29) Tipo Polygonaceae (Fagopyrum), Pb-10368; 30) Corsinipollis sp., Pb-10377; 31) Corsinipollenites sp. 1, Pb-10374; 32) Ulmipollenites sp. 3, Pb-10372; 33) Tipo Betulaceae (Alnus), Pb-10358. 

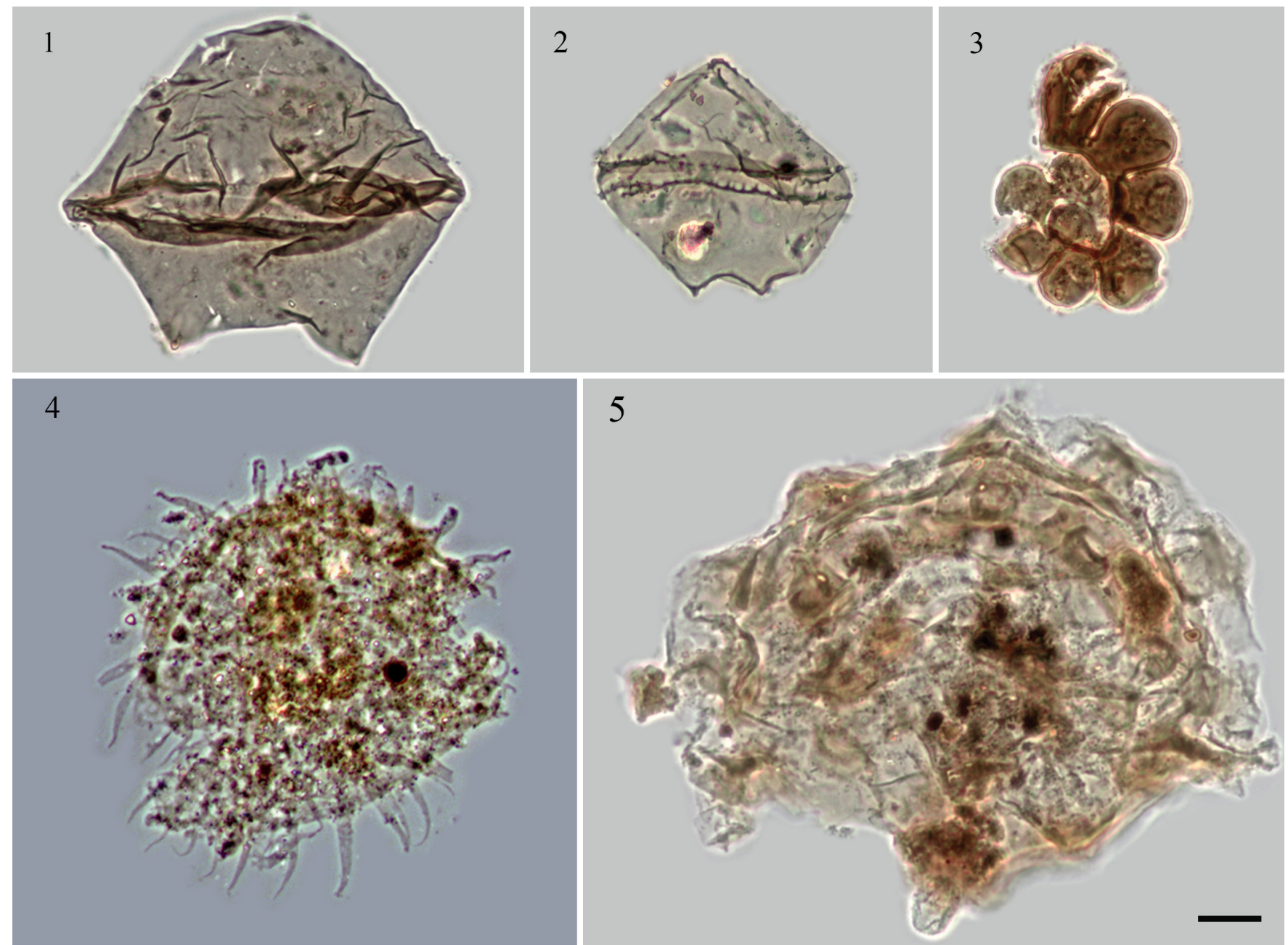

Figura 7 1) y 2) Lejeunecysta sp., Pb-10384 y Pb-10372; 3) testa de microforaminífero, Pb-10383; 4) Lingulodinium sp., Pb-10397; 5) Tuberculodinium aff. vancampoae, $\mathrm{Pb}-10383$.

\section{Discusión}

\subsection{RIQUEZA, DIVERSIDAD DE ESPEGIES Y PALINOZONAS}

El número de taxones continentales en la FSG fue mayor que el de los marinos. Sin embargo, al comparar la riqueza total $(\mathrm{n}=189)$ con los resultados paleopalinológicos obtenidos en otras formaciones geológicas del Oligoceno-Mioceno, se observó que la FSG es particularmente rica. Por ejemplo, en muestras de la formación Weaverville, que aflora en el noreste de California, EEUU, se identificaron 151 taxones, $63 \%$ fueron angiospermas,
$11 \%$ gimnospermas, $13 \%$ esporas de pteridofitas y $11 \%$ algas, acritarcas y dinoflagelados (Barnett, 1982).

En la formación Rancho Berlín del Oligoceno inferior en Chiapas, se encontraron 35 taxones de palinomorfos continentales y marinos (Tomasini-Ortíz y Martínez-Hernández, 1984) y en la formación La Quinta del Oligoceno superior-Mioceno inferior, Biaggi (1978) se registraron 210 taxones; Tomasini-Ortíz y Martínez-Hernández (1984) encontraron 60 y Graham (1999) 29 taxones.

En los depósitos minerales de La Perla del Oligoceno superior-Mioceno, en Chihuahua, se registraron 18 taxones (Corona-Esquivel et al., 2010). 
Tabla 2. Características morfológicas del polen-esporas más abundantes en el conjunto paleopalinológico continental.

\begin{tabular}{|c|c|c|c|c|c|c|}
\hline Taxón & $\begin{array}{c}\text { Eje Ecuatorial } \\
\qquad(\mu \mathrm{m})\end{array}$ & Eje Polar $(\mu \mathrm{m})$ & Abertura & Exina & Estructura & Ornamentación \\
\hline \multicolumn{7}{|l|}{ Esporas } \\
\hline Lusatisporis dettmannae (Drug) Srivastava 1972 & $28.61-4.98$ & - & - & - & - & Granular \\
\hline \multicolumn{7}{|l|}{ Gimnospermas } \\
\hline \multirow{2}{*}{ Pinuspollenites Raatz 1938 ex Potonié 1958} & $\begin{array}{l}\text { Cuerpo central: } \\
42.26-49.62\end{array}$ & $\begin{array}{c}\text { Cuerpo central: } \\
38.83-52.11\end{array}$ & \multirow{2}{*}{ Monosulcado } & \multirow{2}{*}{ Tectada } & Cuerpo central: Columelar & \multirow{2}{*}{ Patrón microrreticulado } \\
\hline & $\begin{array}{l}\text { Sacos aéreos: } \\
\text { 24.64-29.61 }\end{array}$ & $\begin{array}{l}\text { Sacos aéreos: } \\
36.83-43.76\end{array}$ & & & Sacos aéreos: alveolar & \\
\hline Ephedripites Bolkhovitina 1953 & $16.89-48.32$ & $12.31-19.5$ & Pseudosulcado & Tectada & Granular & Psilado \\
\hline $\begin{array}{l}\text { Ephedripites (Distachyapites) claricristatus } \\
\text { (Shakmundes) Krutzsch } 1970\end{array}$ & $32.29-48.16$ & $16.17-21.0$ & $\begin{array}{l}\text { Pseudosulcado con } \\
\text { ramificaciones }\end{array}$ & Tectada & Granular & Psilado \\
\hline Ephedripites aff. krempii Kedves 1977 & $24.2-31.3$ & $13.01-15.79$ & $\begin{array}{c}\text { Pseudosulcado } \\
\text { sin ramificaciones }\end{array}$ & Tectada & Granular & Psilado \\
\hline \multicolumn{7}{|l|}{ Angiospermas } \\
\hline \multicolumn{7}{|l|}{ Monocotiledóneas } \\
\hline Liliacidites Couper 1953 & $19.46-41.15$ & - & Monosulcado & Suctectada & Columelar & $\begin{array}{c}\text { Microrreticulado / Reticulado } \\
\text { heterobrocado }\end{array}$ \\
\hline Graminidites Cookson 1947 ex Potonié 1960 & $18.29-35.66$ & 19.0-33.67 & Monoporado & Tectada & Granular & Psilado \\
\hline \multicolumn{7}{|l|}{ Dicotiledóneas } \\
\hline Chenopodipollis Krutzsch 1966 & $16.49-26.86$ & $17.78-27.77$ & Periporado & Tectado & Columelar & Psilado \\
\hline Tubulifloridites Cookson 1947 ex Potonié 1960 & $12.85-29.21$ & $15.5-34.56$ & $\begin{array}{c}\text { Tricolpado/ } \\
\text { Tricolporado }\end{array}$ & Tectado & Columelar & Equinado \\
\hline
\end{tabular}

Los datos muestran que las formaciones San Gregorio, Weaverville y La Quinta, sobresalen por su elevada riqueza de especies, las cuales registraron valores superiores a 100 taxones. Esto puede deberse a que dichas formaciones se encuentran en paleocuencas marinas, en las cuales uno de los factores tafonómicos comprende el proceso de depósito de palinomorfos que incluye tanto el aporte de elementos terrestres, como marinos; mientras que en las paleocuencas continentales solo incluyen los taxones continentales. Palafox-Silva (2013) menciona que la cantidad de palinomorfos continentales está determinada por el aporte eólico, fluvial y la escorrentía; mientras que la cantidad de palinomorfos marinos se vincula con la productividad marina en las zonas costeras y su transporte desde las partes someras de la cuenca a las más profundas.

La diversidad de especies a $\left(H^{\prime}\right)$ y la uniformidad $\left(\mathcal{F}^{\prime}\right)$ del conjunto continental en cada una de las muestras del barreno B4 (Tabla 1), presentaron valores menores que aquellos registrados en otras paleocuencas continentales del Cenozoico de México. Tal es el caso de la formación Tehuacán del Mioceno medio (Ramírez-Arriaga et al., 2014a), localizada en Puebla, en la cual la diversidad de especies a $\left(H^{\prime}\right)$ varió entre 1.3 a 2.8 en cada una de las muestras, pero se mantiene más o menos constante entre 2 y 2.8. Además, la distribución de individuos dentro de cada taxón $\left(\mathcal{F}^{\prime}\right)$ fue casi homogénea en la mayoría de las muestras entre 0.8 a uno. Sin embargo, si se revisa la diversidad de especies a $\left(H^{\prime}\right)$ del conjunto total del barreno B4, se observa que los valores más altos de diversidad de especies son muy semejantes a los de la formación Tehuacán, entre 2.8 y 2.9, pero son más variables ya que van de 0 a 2.9 con una uniformidad de entre 0 y 1 .

De las tres palinozonas definidas en el barreno B4, la zona $\mathrm{B}$ presentó mayor riqueza de taxones $(\mathrm{Pb}-$ 10384 a Pb-10365) (Figura 4). Como consecuencia, en este intervalo se encuentran las muestras que tuvieron la diversidad a $\left(H^{\prime}\right)$ más alta $(>2)$ del conjunto de continental (Pb-10368, Pb-10369, Pb10372, Pb-10374, Pb-10378, Pb-10379, Pb-10381 y $\mathrm{Pb}-10382)$ (Figura 2).

Los análisis de diversidad de especies a $\left(H^{\prime}\right)$ y uniformidad $\left(\mathcal{F}^{\prime}\right)$, son medidas que en conjunto permiten definir el número de especies presentes en cada muestra y, además, cómo se reparten los palinomorfos dentro de cada especie. Los valores de los índices obtenidos en las muestras del barreno 
B4 son importantes, ya que permitirán hacer comparaciones con resultados palinoflorísticos que se obtengan en estudios posteriores de la FSG, o en otras paleocuencas que correspondan al Oligoceno superior-Mioceno inferior en México.

\subsection{AFINIDADES BIOLÓGICAS}

Algunos de los elementos continentales del barreno B4, tienen afinidad botánica con familias, géneros o especies actuales.

De los taxones que dominaron el conjunto continental en la zona B, Chenopodipollis se relaciona con miembros de las familias Amaranthaceae-Chenopodiaceae. Graminidites, se vincula con géneros actuales de la familia Poaceae y Liliacidites con las familias Liliaceae, Bromeliaceae y Arecaceae. $\mathrm{Lu}^{-}$ satisporis dettmannae es una espora de pteridofitas que tiene afinidad botánica con Selaginella oregana (Srivastava, 1972).

Los taxones registrados en menor cantidad en la zona $\mathrm{B}$, presentan afinidad botánica en los casos de: Corsinipollenites que se relaciona con miembros actuales de la familia Onagraceae y Corsinipollis que tiene afinidad botánica con el género Ludwigia (Onagraceae), el cual tiene varias especies que se desarrollan principalmente en lugares húmedos (Ramírez-Arriaga et al., 2014b). En la actualidad, la familia Onagraceae está bien representada en el norte y sur de América, y en particular en México, en donde presenta un alto porcentaje de endemismos (Miguel-Vázquez y Cerros-Tlatilpa, 2013).

Otras formas-géneros registradas en el barreno B4 se asocian con géneros y familias actuales, por ejemplo: Ephedripites (Ephedra), Rugulitriporites (Bursera), Momipites coryloides y M. tenuipolus (Engelhardtia, Alfaroa y Oreomunnea), Caryapollenites (Carya) Quercoidites (Quercus), Pinuspollenites (Pinus), Ulmipollenites (Ulmus/Zelkova), Fraxinoipollenites (Fraxinus), Leguminosaepites, Psilatricolpites y Psilatricolporites (Leguminosae), Margocolporites (Caesalpiniaceae), Malpighiaceoidites (Malpighiaceae), Striatricolporites y Retitricolporites (Anacardiaceae), cf. Camarazonosporites y Lycopodiacidites (Lycopodium), Cicatricosisporites (Anemia y Mohria), Deltoidospora (Cyatheaceae, Dicksoniaceae y Schizaeaceae), Undulatisporites
(Ophioglossaceae), Polypodiisporites (Polypodiaceae) y Stereisporites (briofitas).

$\mathrm{El}$ género Tubulifloridites sobresalió principalmente en la zona $\mathrm{C}$ del barreno (Pb-10360-Pb-10358) (Figura 4). Se identificaron cuatro tipos de Tubulifloridites, los cuales se diferenciaron entre ellos por el tamaño y número de las espinas. Su morfología los relaciona con miembros de la familia Asteraceae, en especial, con las tribus Astereae, Eupatorieae and Heliantheae (Galván-Escobedo et al., 2015a). Graham (1996), se refiere al polen fósil de Asteraceae como tipo AHH et al., en el cual incluye polen fósil similares a los de las tribus Astereae, Heliantheae y Heleniae.

\subsection{PALEOVEGETACIÓN}

Los requerimientos ecológicos de los taxones cuya afinidad biológica se corresponde a los elementos continentales registrados en el barreno B4, permiten sugerir el tipo de vegetación en el que pudieron estar presentes dichos elementos. Los tipos de vegetación que se sugieren, son de acuerdo a la clasificación propuesta por Rzedowski (2006).

El elemento más abundante en el barreno B4, Chenopodipollis (Amaranthaceae-Chenopodiaceae) se asocia con condiciones semisecas. Es probable que debido al incremento de las temperaturas que se registró en el Oligoceno superior (Zachos et al., 2001), este taxón haya llegado a convertirse en un elemento común en la región de La Purísima. El abrupto descenso de la temperatura a finales del Oligoceno superior (Zachos et al., 2001) pudo haber sido desfavorable para este taxón, ya que en paleocuencas del Neógeno del centro de México, se registra en frecuencias más bajas que en la FSG (Martínez-Hernández y Ramírez-Arriaga, 1999; Ramírez-Arriaga et al., 2006; Ramírez-Arriaga et al., 2014a).

Las formas-géneros que son afines a Amaranthaceae-Chenopodiaceae, Anacardiaceae Rhus-, Arecaceae, Asteraceae, Bromeliaceae, Caesalpiniaceae, Euphorbiaceae, Leguminosae, Liliaceae, Malpighiaceae, Moraceae, Onagraceae, Poaceae, Polemoniaceae, Sterculiaceae, Bursera, Celtis, y Ephedra, son familias o géneros que coe- 
xisten y son elementos importantes en el bosque tropical caducifolio (BTC), caracterizado por una época de seca larga (Rzedowski y Calderón de Rzedowski, 1987; Becerra, 2005; Rzedowski, 2006; Miranda y Hernández-X., 2014).

La distribución actual del BTC se advierte en la vertiente pacífica de México, desde Sonora hasta Yucatán, en el Bajío, en la cuenca del Balsas y en la cuenca del Papaloapan, entre los 0 y 1700 m de altitud, en zonas con clima caliente subhúmedo con lluvias en verano (Aw), templado subhúmedo con lluvias en verano $(\mathrm{Cw})$ y seco $(\mathrm{Bs})$, con temperaturas promedio anual de 20 a $29^{\circ} \mathrm{C}$, con precipitación promedio anual de 300 a $1200 \mathrm{~mm}$ y con una temporada de secas que dura alrededor de cinco a ocho meses, por lo que la gran mayoría de las especies pierden sus hojas durante esta temporada (Rzedowski y Calderón de Rzedowski, 1987; Rzedowski, 2006; Challenger y Soberón, 2008; Miranda y Hernández-X., 2014).

El BTG en Baja California Sur, se encuentra en la actualidad en las partes inferiores y medias de las sierras de La Laguna y La Giganta (Rzedowski, 2006; León de la Luz et al., 2012). Este ecosistema ha sido reconocido en varias localidades del Paleógeno/Neógeno en el centro de México (Martínez-Hernández y Ramírez-Arriaga, 1999; Ramírez-Arriaga, 2005; Martínez-Hernández y Ramírez-Arriaga, 2006). Los estudios sobre el origen y expansión del BTC, con base en la diversificación y cambios espaciales del género Bursera (Becerra, 2005), indican que este bosque se estableció hace 30 a 20 millones de años, primero en el oeste del país, incluyendo a la península de Baja California, y después se expandió hacia el sur y oeste del territorio, y tenía una distribución mucho más amplia de la que tiene en la actualidad.

Por otro lado, Momipites (Engelhardtia, Alfaroa y Oreomunnea) y algunos taxones de polen fósil registrados en el barreno B4, se corresponden con elementos florísticos actuales que coexisten en el bosque mesófilo de montaña (BMM) (Puig et al., 1987; Graham y Dilcher, 1995; Sánchez-Rodríguez et al., 2003; Rzedowski, 2006; Challenger y Soberón, 2008; Baab et al., 2010; Álvarez-Zuñiga et al., 2012). Tales elementos corresponden a géneros como Alnus, Carya, Fraxinus, Quercus, Ulmus/ Zelkova, y algunos otros a las familias Betulaceae, Leguminosae, Melastomataceae, Salicaceae y Tiliaceae, así como la presencia las diferentes esporas de afinidad con Anemia, Lycopodium, Mohria, Selaginella, Cyatheaceae, Dicksoniaceae y Schizaeaceae, Ophioglossaceae, Polypodiaceae y briofitas.

Es notorio mencionar que la gran mayoría de los granos de polen mencionados en el párrafo anterior son géneros o familias de afinidad boreal (excepto Leguminosae y Melastomataceae) y, que además, son elementos arbóreos y fisionómicamente importantes que en la actualidad coexisten como componentes del BMM (Challenger, 1998). Los elementos encontrados pertenecientes a las familias Leguminosae y Melastomataceae son de afinidad neotropical y son componentes del estrato arbustivo en el sotobosque del BMM (Challenger, 1998).

Al BMM de México se le localiza sobre los sistemas montañosos del país con elevada humedad atmosférica. Este bosque se distribuye sobre una angosta franja altitudinal donde prevalecen concentraciones de nubes y neblina (Hamilton et al., 1995), copiosa precipitación pluvial conjuntamente con elevada condensación de agua y bajas tasas de evapotranspiración de la vegetación (Bruijnzeel et al., 1993). A lo largo de la distribución natural del BMM prevalecen los climas templado húmedo con lluvias todo el año (Cf), caliente húmedo con lluvias todo el año (Af), caliente húmedo con lluvias en verano (Am), caliente subhúmedo con lluvias en verano (Aw) y templado subhúmedo con lluvias en verano $(\mathrm{Cw})$, con temperatura media anual entre 12 y $23^{\circ} \mathrm{C}$ y precipitaciones que fluctúan entre 1000 y $5800 \mathrm{~mm}$ anuales, siendo la norma de aproximadamente $2000 \mathrm{~mm}$ anuales. La distribución actual del BMM en México es discontinua, a manera de un archipiélago de fragmentos de bosque sobre las principales cadenas montañosas entre los 1500 y $2500 \mathrm{msnm}$ (Challenger y Soberón, 2008; López-Mata et al., 2012), pero se le puede localizar ocasionalmente a los 400 m de altitud (Rzedowski, 2006). 
Rzedowski (1996) menciona que el BMM pudo haber tenido una distribución más amplia en el Paleógeno/Neógeno, por lo que los fragmentos que se localizan en los estados de Oaxaca, Chiapas e Hidalgo (Álvarez-Zuñiga et al., 2012), Tamaulipas, San Luis Potosí, Puebla, Veracruz, Sinaloa, Nayarit, Jalisco, Michoacán y Guerrero (Rzedowski, 2006; Baab et al., 2010), son considerados como relictos de este tipo de bosque.

Aunque en la actualidad no existe registro de la presencia de BMM en la península de Baja California, los palinomorfos registrados en el barreno $\mathrm{B} 4$ de la presente investigación, sugieren que durante el Oligoceno superior-Mioceno inferior, algunos elementos importantes con afinidad botánica a los del actual BMM, pudieron haberse desarrollado en regiones adyacentes a la zona de depósito del barreno B4. Cabe recordar que, durante el Oligoceno superior-Mioceno inferior, la península de Baja California se encontraba unida al continente haciendo contacto con los estados de Sinaloa, Nayarit y Jalisco, a la altura de Cabo Corrientes (20²4'32'" N y $105^{\circ} 41^{\prime} 40^{\prime \prime}$ O) en donde el BMM se encontraba ampliamente distribuido (Rzedowski, 1996).

En el barreno B4, se registró polen con afinidad botánica a Quercus y Pinus en muy bajas frecuencias (Quercoidites < 1-10 \% y Pinuspollenites 2-16\%). Los bosques de Quercus y Pinus, existen en la actualidad en la penínsulas de Baja California y casi en todo el país, excepto en la península de Yucatán (Rzedowski, 2006). Los estudios de lluvia de polen actual en bosques de Pinus, indican que este género suele registrarse en porcentajes muy elevados, entre 34 y 57, mientras que el polen de Quercus, está presente hasta en un $25 \%$ (Ortega-Rosas et al., 2008; Velázquez-Silva, 2008). En la lluvia de polen del BMM, Pinus se encuentra en porcentajes muy bajos (6.1 \%), al igual que el de Quercus $(7.6 \%)$ (Palacios-Chávez y Rzedowski, 1993). De acuerdo con esta información, es posible que los registros de Quercoidites y Pinuspollenites del barreno B4, más que representar un bosque de Quercus y Pinus, podrían haber formado parte del BMM, tal y como se ha encontrado en este tipo de vegetación actualmente (Luna-Vega et al., 1994; Rzedowski, 2006).

\subsection{AMBIENTE DE DEPÓSITO}

El índice de palinomorfos marinos, aportó información sobre las condiciones paleoecológicas y paleoclimáticas en el que tuvo lugar el proceso de sedimentación del barreno B4.

La presencia de palinomorfos marinos tales como dinoflagelados, microforaminíferos, escolecodontos y algas, se asocia con ambientes marinos neríticos cercanos a la costa (Tomasini-Ortíz y Martínez-Hernández, 1984). En ambientes de depósito transicionales a neríticos, la diversidad de dinoflagelados se incrementa al ascender el nivel del mar y disminuye en los estadios regresivos (Narváez y Helenes-Escamilla, 2003). Así mismo, se ha detectado una relación directa entre altos valores de diversidad de dinoflagelados y estabilidad ambiental en el océano (Palafox-Silva, 2013).

La riqueza de dinoflagelados Gonyaulacales en el barreno B4, fue igual que la de los Peridiniales. Sin embargo, las frecuencias de individuos en cada género, fue mayor en los del orden Gonyaulacales $(\mathrm{n}=272)$ que en los del orden Peridiniales $(\mathrm{n}=20)$. Esto puede deberse a que los primeros suelen registrarse de manera más abundante en zonas tropicales (Narváez y Helenes-Escamilla, 2003; Helenes-Escamilla, 2004) como es el caso de nuestro sitio de estudio pero, además, a que el tratamiento con acetólisis de las muestras pudo influir en la eliminación de dinoflagelados Peridiniales (Kodrans-Nsiah et al., 2008; Zonneveld et al., 2008), por lo que es probable que el conjunto de estos organismos no esté representado a cabalidad. Una mayor diversidad de dinoflagelados con predominio de taxones Gonyaulacales (los cuales en su mayoría son autotróficos), se relaciona con aguas oceánicas cálidas y con facies marinas someras (Martínez-Hernández et al., 1980; Helenes-Escamilla, 2004; Ramírez-Arriaga et al., 2012a; Palafox-Silva, 2013). En este sentido, los géneros Polysphaeridium y Tuberculodinium registrados en el barreno B4, son considerados taxones 
característicos de ambientes transicionales, como por ejemplo estuarios, lagunas costeras o escorrentías (Martínez-Hernández y Hernández-Campos, 1991; Pross y Brinkhuis, 2005), Cleistosphaeridium, Lingulodinium, Operculodinium y Spiniferites, son taxones que suelen desarrollarse en ambientes marinos neríticos cercanos a la costa (Pross y Brinkhuis, 2005; Sluijs et al., 2005).

\section{Conclusiones}

Los registros de elementos continentales sugieren que durante el Oligoceno superior - Mioceno inferior, se pudieron desarrollar tipos de vegetación con requerimientos ecológicos semejantes a los que tienen el bosque tropical caducifolio y el bosque mesófilo de montaña en la actualidad.

Los elementos continentales que sobresalieron por su abundancia en el barreno B4 (Chenopodipollis, Graminidites, Liliacidites y Tubulifloridites), y otros taxones que se registraron con menores frecuencias (Corsinipollenites, Corsinipollis, Ephedripites, Malpighiaceoidites, Margocolporites, Psilatricolpites, Psilatricolporites, Rugulitriporites, tipos Anacardiaceae -Rhus-, Euphorbiaceae, Moraceae, Polemoniaceae, Sterculiaceae y Celtis), son afines a elementos que coexisten hoy en día en el bosque tropical caducifolio. Los taxones tales como Alnus, Caryapollenites, Fraxinoipollenites, Momipites, Quercoidites, Ulmipollenites, tipos Leguminosae, Melastomataceae, Salicaceae y Tiliaceae, así como la presencia las diferentes esporas de afinidad con Anemia, Lycopodium, Mohria, Selaginella, Cyatheaceae, Dicksoniaceae y Schizaeaceae, Ophioglossaceae, Polypodiaceae y briofitas, se relacionan con elementos florísticos actuales que cohabitan en el bosque mesófilo de montaña.

Es posible que, a nivel local, en la zona de depósito del barreno B4, durante el Oligoceno superior - Mioceno, predominara un clima semiseco y cálido, el cual favoreció el crecimiento de formas vegetales afines a los elementos florísticos del bosque tropical caducifolio. A nivel regional, en las zonas de mayor altitud, es probable que se hubiera de- sarrollado un bosque mesófilo de montaña, sobre todo si se considera la cercanía de la península de Baja California con los territorios de los estados de Sinaloa, Nayarit y Jalisco, en donde este bosque estaba bien representado en las zonas altas y húmedas.

El Índice de Palinomorfos Marinos, así como el registro de los elementos continentales y los dinoflagelados de los géneros Cleistosphaeridium, Lingulodinium, Operculodinium, Polysphaeridium, Spiniferites y Tuberculodinium, corroboran que el barreno B4 se depositó en un ambiente transicional a nerítico, relativamente cercano a la costa.

Las formas de polen con importancia cronoestratigráfica: Corsinipollenites (Onagraceae) (Maastrichtiano-Mioceno medio), Ephedripites claricristatus (Ephedraceae) (Eoceno medio - Oligoceno tardío/ Oligoceno temprano - Mioceno medio), Momipites coryloides (Paleoceno temprano - Mioceno medio) y M. tenuipolis (Paleoceno temprano-Oligoceno temprano), se presentaron en bajas frecuencias, lo que dificulta hacer inferencias sobre su significado cronoestratigráfico en el barreno B4. Los intervalos estratigráficos de los dinoflagelados Tuberculodinium aff. vancampoae (Oligoceno superior - hasta la actualidad) y Spiniferites pseudofurcatus (Maastrichtiano - Mioceno medio), indican que los estratos del barreno B4 debieron haberse depositado entre el Oligoceno superior y el Mioceno medio, sin poderse asignar una edad con mayor resolución.

\section{Agradecimientos}

A los proyectos de la UNAM: PAPIIT-IN105411 "Evolución de la flora y cambios climáticos en cuencas terciarias marinas y continentales de México" y PAPIIT-IN114914 "Cambios palinoflorísticos y evolución de la vegetación a través del Paleógeno-Neógeno en Baja California Sur y centro de México" por el financiamiento. Al CONACyT por la beca de doctorado 216587, y al Colegio de Posgraduados Campus Montecillo por el apoyo a la primera autora para el desarrollo de su investigación doctoral "Palinoestratigrafía 
de las Fosforitas de Baja California Sur, México y Reconstrucción de la Vegetación durante el Oligoceno-Mioceno". A la Dra. Ana Luisa Carreño del Instituto de Geología de la UNAM, por la donación del barreno B4. A los Drs. Javier Helenes Escamilla, Lauro López Mata, Enrique Martínez Hernández y a los revisores anónimos, por sus comentarios, recomendaciones y sugerencias que ayudaron a mejorar este artículo.

\section{Referencias}

Alatorre, A.E., 1988, Stratigraphy and depositional environments of the phosphorite-bearing Monterrey Formation in Baja California Sur: Economic Geology, 83(8), 1918-1930.

Álvarez-Zúñiga, E., Sánchez-González, A., López-Mata, L., Tejero-Diez, J.D., 2012, Composición y abundancia de las pteridofitas en el bosque mesófilo de montaña del municipio de Tlanchinol, Hidalgo, México: Botanical Sciences, 90(2), 163-177.

Applegate, S.P., 1986, The El Cien Formation: Strata of Oligocene and early Miocene age in Baja California Sur: Universidad Nacional Autónoma de México, Instituto de Geología, Revista, 6(2), 145-162.

Aranda-Gómez, J.J., Henry, C.D., Luhr, J.F., 2000, Evolución tectonomagmática postpaleocénica de la Sierra Madre Occidental y de la porción meridional de la provincia tectónica de Cuencas y Sierras, México: Boletín de la Sociedad Geológica Mexicana, 53, 59-71.

Baab, K., Blanco y Correa, M., González-Flores, R.E., Navarrete-Carrillo, J.A., Téllez-Valdés, O., Vega-Aviña, R., 2010, II. Serranías de Nayarit, en Toledo-Aceves, T. (ed.), El bosque mesófilo de montaña en México: Amenazas y oportunidades para su conservación y manejo sostenible: México, Comisión Nacional para el Conocimiento y Uso de la Biodiversidad, 50-59.
Barnett, J., 1982, Palynology and paleoecology of Weaverville Formation (Tertiary): Seattle, University of Washington, Tesis de maestría en ciencias, $118 \mathrm{p}$.

Beal, C.H., 1948, Reconnaissance of the geology and oil possibilities of Baja California, en The Geological Society of America, Memoir 31, 138.

Becerra, J.X., 2005, Timing the origin and expansion of the Mexican tropical dry forest: Proceedings of the National Academy of Sciences of the United States, 102(31), 10919-10923.

Biaggi, R.E., 1978, Palynology and Paleoecology of some Oligo-Miocene sediments from Chiapas, Mexico: California, U.S.A, Walla Walla College California, Tesis de Maestría en Ciencias, 92 p.

Bruijnzeel, L.A., Waterloo, M.J., Proctor,J., Kuiters, A.T., Kotterink, B., 1993, Hydrological observations in montane rain forests on Gunung Silam, Sabah, Malaysia, with special reference to the 'Massenerhebung' effect: Journal of Ecology, 81, 141-167.

Carrasco-Velázquez, B.E., Martínez Hernández, E., Ramírez-Arriaga, E., 2009, Estratigrafía de la Formación El Morro del PaleocenoEoceno en Zimapán, Hidalgo: Boletín de la Sociedad Geológica Mexicana, 61(3), 403-417.

Carreño, A.L., Helenes, J., 2002, Geology an Ages of the Islands, en Case, T., Cody, M.L., Ezcurra, E. (eds.), A new Island Biogeography of the Sea of Cortes: New York. U.S.A., Oxford University Press, 14-40.

Carreño, A.L., Smith, J.T., 2007, Stratigraphy and correlation for the ancient Gulf of California and Baja California Peninsula, Mexico: New York, U.S.A., Paleontological Research Institution, Bulletin of American Paleontology, 371, 146 p. 
Carrillo-Berumen, R., Quattrocchio, M.E., Helenes-Escamilla, J., 2013, Palinomorfos continentales del Paleógeno de las formaciones Chorrillo Chico y Agua Fresca, Punta Prat, Región de Magallanes, Chile: Andean Geology, 40(3), 539-560.

Challenger, A., 1998, Utilización y conservación de los ecosistemas terrestres de México. Pasado, presente y futuro: México, CONABIOInstituto de Biología, UNAM-Agrupación Sierra Madre, 847 p.

Challenger, A., Soberón, J., 2008, Los Ecosistemas Terrestres, en Soberón, J., Halffter, G., Llorente-Bosques, J. (eds.), Capital Natural de México: México, 1, Conocimiento actual de la diversidad, CONABIO, 87-108.

Corona-Esquivel, R., Martínez-Hernández, E., Henríquez, F., Olov-Nyström, J., Tritlla, J., 2010, Palynologic evidence for iron-oxide ash fall at La Perla, an Oligocene Kirunatype iron ore deposit in northern Mexico: GFF (Geologiska föreningen), 132, 137-181.

Darton, N.H., 1921, Geologic reconnaissance in Baja California: Journal of Geology, 29, 720-748.

Donoghue, M.J., 2008, A phylogenetic perspective on the distribution of plant diversity: Proceedings of the National Academy of Sciences of the United States of America, 105, Supplement 1, 11549-11555.

Fischer, R., Galli-Olivier, C., Gidde, A., Schwennicke, T., 1995, The El Cien Formation of southern Baja California, Mexico: Stratigraphic precisions: Newsletters on Stratigraphy, 32(3), 137-161.

Galli-Olivier, C., 1993, Fosforitas de San Juan de la Costa (Bahía de la Paz), Baja California Sur, México: 17 años de investigaciones: Revista de Investigación Científica, 4(1), 115-135.

Galván-Escobedo, I.G., Ramírez-Arriaga, E., Reyes-Salas, M., Martínez-Hernández, E., Ángeles-García, S., García-Moya, E., 2015a, Descriptions and botanical affinities of Tubulifloridites Cookson 1947 ex Potonié 1960 pollen grains from the San Gregorio Formation, Baja California Sur, Mexico: Acta Microscópica, 24(1), 7-15.

Galván-Escobedo, I.G., Ramírez-Arriaga, E., Martínez-Hernández, E., Reyes-Salas, M., Ángeles-García, S., Kohashi-Shibata, J., 2015b, Morphological descriptions of late Oligocene - early Miocene spores: San Gregorio Formation, Baja California Sur, Mexico: Acta Microscópica, 24(1), 41-52.

Graham, A., 1976, Studies in neotropical paleobotany. II. The Miocene Communities of Veracruz, México: Annals of Missouri Botanical Garden, 63, 787-842.

Graham, A., 1996, Contribution to the geologic history of the Compositae, en Hind, D.J.N., Beentje, H.J. (eds.), Compositae: Systematics Proceedings of the International Compositae Conference: Kew, 1994, 1, 123-140.

Graham, A., 1999, Studies in Neotropical paleobotany. XIII. An Oligocene-Miocene palynoflora from Simojovel, Chiapas, México: American Journal of Botany, 86, $17-31$.

Graham, A., Dilcher, D., 1995, The Cenozoic record of tropical dry forest in northern Latin America and the southern United States, en Bullock, S.H., Mooney, H.A., Medina, E. (eds.), Seasonally dry tropical forests: Gran Bretaña, Cambridge University, 124-145.

Grimm, E.C., 1987, CONISS: A Fortran 77 program for stratigraphically constrained cluster analysis by the method of incremental sum of squares: Computer \& Geosciences, 13(1), 13-35.

Grimm, E.C., 201 1, Tilia Software, Versión 1.7.16: Springfield, IL., Illinois State Museum.

Grimm, K.A., Föllmi, K.B., 1994, Doomed Pioneers: Allochthonous Crustacean Tracemakers in Anaerobic Basinal Strata, Oligo-Miocene San Gregorio Formation, Baja California Sur, Mexico: Palaios, 9, 313-334. 
Hamilton, L.S., Juvik, O.J., Scatena, F.N., 1995, The Puerto Rico tropical cloud forest symposium: Introduction and workshop synthesis, en Hamilton, L.S., Juvik, O.J., Scatena, F.N. (eds.), Tropical Montane Cloud Forest: Nueva York, Springer-Verlag, Ecological Studies 110, 1-23.

Hausback, B.P., 1984, Cenozoic Volcanic and Tectonic Evolution of Baja California Sur, México, en Frizzell, I. (ed.), Geology of the Baja California Peninsula: Los Angeles, California, U.S.A., Pacific Section, Society of Economic Paleontologists and Mineralogists, 219-236.

Heim, A., 1922, Notes on the Tertiary of southern Lower California: Geological Magazine, 59, 529-547.

Helenes-Escamilla, J., 2004, Dinoestratigrafía Tropical del Terciario: Boletín de la Asociación Mexicana de Geólogos Petroleros, $\mathrm{LI}(1), 77-79$.

Helenes-Escamilla, J., Carreño, A.L., 1999, Neogene sedimentary evolution of Baja California in relation to regional tectonics: Journal of South American Earth Sciences, 12, 589-605.

Helenes-Escamilla, J., De Guerra, C., Vasquez, J., 1998, Palynology and chronostratigraphy of the upper Cretaceous in the subsurface of the Barinas area, western Venezuela: Bulletin of the American Association of Petroleum Geologists, 82(7), 1308-1327.

Helenes-Escamilla, J., Carreño, A.L., CarrilloBerumen, R.M., 2009, Middle tolate Miocene chronostratigraphy and development of the northern Gulf of California Marine Micropaleontology, 72(1-2), 10-25.

Kim, W.H., Barron, J.A., 1986, Diatom biostratigraphy of the upper Oligocene to lowermost Miocene San Gregorio Formation, Baja California Sur, Mexico: Diatom Research, 1(2), 169-187.

Kodrans-Nsiah, M., de Lange, G.J., Zonneveld, K.A.F., 2008, A natural exposure experiment on short-term species-selective aerobic degradation of dinoflagellate cysts: Review of Palaeobotany and Palynology, 152(1-2), 32-39.

Langenheim, J.H., Hackner, B.L., Bartlett, A., 1967, Mangrove pollen at the positional site of Oligocene-Miocene amber from Chiapas, Mexico: Botanical Museum Leaflets, Harvard University, 21, 289-324.

Ledesma-Vázquez,J., Carreño, A.L., 2010, Origin, Age, and Geological Evolution of the Gulf of California, en Brusca, R.C. (ed.), The Gulf of California. Biodiversity and Conservation: Arizona, U.S.A., The University of Arizona Press and The Arizona-Sonora Desert Museum, 7-23.

León de la Luz, J.L., Domínguez-Cadena, R., Mendel-Narváez, N., 2012, Florística de la Selva Baja Caducifolia de la Península de Baja California, México: Botanical Sciences, 90(2), 143-162.

López-Mata, L., Villaseñor, J.L., Cruz-Cárdenas, G., Ortíz, E., Ortíz-Solorio, C., 2012, Predictores ambientales de la riqueza de especies de plantas del Bosque Húmedo de Montaña de México: Botanical Sciences, 90(1), 27-36.

Luna-Vega, I., Ocegueda-Cruz, S., AlacántaraAyala, O., 1994, Florística y notas biogeográficas del bosque mesófilo de montaña en el municipio de Tlanchinol, Hidalgo, México: Anales del Instituto de Biología, Universidad Nacional Autónoma de México, Serie Botánica, 65(1), 31-62.

Magurran, A.E., 1989, Diversidad ecológica y su medición: Barcelona, España, Editorial Vedra, $200 \mathrm{p}$.

Martín-Barajas, A., 2000, Volcanismo y extensión en la Provincia Extensional del Golfo de California: Boletín de la Sociedad Geológica Mexicana, 53, 72-83.

Martínez-Hernández, E., 1991, Palinología de carbones del Plioceno en Zacultipan, Hidalgo (resumen), en Memorias del Primer Congreso Mexicano de Mineralogía, Pachuca, Hidalgo, 98-101. 
Martínez-Hernández, E., 1992a, Caracterización ambiental del Terciario de la región de Ixtapa, estado de Chiapas- un enfoque palinoestratigráfico: Universidad Nacional Autónoma de México, Instituto de Geología, Revista, 10, 54-64.

Martínez-Hernández, E., 1992b, La Vegetación y el clima de México: Historia del Cretácico al Terciario, basada en evidencias palinológicas: Ciencia, 43(número especial), 69-72.

Martínez-Hernández, E., Tomasini-Ortíz, A.C., 1986, Palinoestratigrafía preliminar del Terciario continental de Sonora (resumen), en Primer Simposio de Geología Regional de México, Instituto de Geología, Giudad Universitaria, 19-21.

Martínez-Hernández, E., Hernández-Campos, H., 1991, Distribución de quistes de dinoflagelados y acritarcas en sedimentos Holocénicos del Golfo de California: Paleontología Mexicana, 57, 1-133.

Martínez-Hernández, E., Ramírez-Arriaga, E., 1996, Oligocene phosphorite sequence and presence of low biomass vegetation from San Gregorio Fm., Baja California, Mexico (resumen), en IX International Palynological Congress, Houston, Texas, U.S.A., 98.

Martínez-Hernández, E., Ramírez-Arriaga, E., 1999, Palinoestratigrafía de la región de Tepexi de Rodríguez, Puebla, México. Implicaciones Cronoestratigráficas: Revista Mexicana de Ciencias Geológicas, 16(2), 187-207.

Martínez-Hernández, E., Ramírez-Arriaga, E., 2006, Tertiary Palynofloristic correlations between Mexican Formations with emphasis in dating the Balsas Group, en Vega, F., Nyborg, T.G., Perrilliat, M.C., MontellanoBallesteros, M., Cevallos-Ferriz, S., QuirozBarroso, S.A. (eds.), Studies on Mexican Paleontology: Dordrecht, The Netherlands, Springer, 19-45.

Martínez-Hernández, E., Hernández-Campos, H., Sánchez-López, M., 1980, Palinología del Eoceno en el Noreste de México: Universidad Nacional Autónoma de México. Instituto de Geología, Revista, 4(2), 155-166.

Miguel-Vázquez, M.I., Cerros-Tlatilpa, R., 2013, Onagraceae de Morelos, México: Revista Mexicana de Biodiversidad, 84(4), 1309-1315.

Miranda, F., Hernández-X., E., 2014, Los tipos de vegetación de México y su clasificación: México, D.F., Fondo de Cultura Económica, Sociedad Botánica de México, Comisión Nacional para el Conocimiento y Uso de la Biodiversidad, $220 \mathrm{p}$.

Narváez, Y., Helenes-Escamilla, J., 2003, Dinoflagelados fósiles y nanofósiles calcáreos: utilidad bioestratigráfica en la exploración de petróleo: Boletín de la Sociedad Geológica del Perú, 96, 99-105.

Ortega-Rosas, G.I., Peñalba, M.G., LópezSáenz, J.A., Van Devender, T.R., 2008, Retrospectiva del bosque de pino y encino de la sierra madre occidental, Sonora, noroeste de México, hace 1000 años: Acta Botánica Mexicana, 83, 69-92.

Palacios-Chávez, R., Rzedowski, J., 1993, Estudio palinológico de las Floras Fósiles del Mioceno inferior y principio del Mioceno medio de la Región de Pichucalco, Chiapas, México: Acta Botánica Mexicana, 24, 1-96.

Palafox-Silva, L.H., 2013, Registro palinológico en sedimentos laminados de la cuenca La Paz, Baja California Sur y su relación con cambios paleoceanográficos y paleoclimáticos: Ensenada, Baja California, México, Centro de Investigación Científica y de Educación Superior de Ensenada, Tesis de Maestría, 80 p.

Pielou, E.G., 1975, Ecological Diversity: New York, John Wiley \& Son, 165 p.

Pross, J., Brinkhuis, H., 2005, Organic-walled dinoflagellate cysts as paleoenvironmental indicators in the Paleogene; a synopsis of concepts: Paläontologische Zeitschrift, 79(1), $53-59$. 
Puig, H., Bracho, R., Sosa, V., 1987, El Bosque Mesófilo de Montaña: Composición Florística y Estructura, en Puig, H., Bracho, R. (eds.), El Bosque Mesófilo de Montaña de Tamaulipas: México, D.F., Instituto de Ecología, A.C., 55-80.

Quiroz-García, L., Martínez-Hernández, E., 1987, Conjuntos palinológicos recuperados de los lignitos del Plioceno, Tlacolulan, Veracruz (resumen), en $\mathrm{X}$ Congreso Mexicano de Botánica, Guadalajara, Jalisco, Contribución 418.

Ramírez-Arriaga, E., 2005, Reconstrucción Paleoflorística de la Formación Cuayuca con base en análisis palinoestratigráfico e implicaciones paleogeográficas: México, D.F., Universidad Nacional Autónoma de México, Tesis doctoral, $231 \mathrm{p}$.

Ramírez-Arriaga, E., Reyes-Salas, M., 2014, Pollen morphology of the Momipites group (Juglandaceae) recovered in Cenozoic Basins from central Mexico and Baja California Sur: Acta Microscópica, 23(2), 101-110.

Ramírez-Arriaga, E., Prámparo, M., MartínezHernández, E., Valiente-Banuet, A., 2006, Palynology of the Paleogene Cuayuca Formations (stratotype sections), southern Mexico: Chronostratigraphical and palaeoecological implications: Review of Paleobotany and Palynology, 141, 259-275.

Ramírez-Arriaga, E., Martínez-Hernández, E., Flores-Olvera, H., Ochotorena, H., Prámparo, M., 2008, Correlation of the Late Eocene-Early Oligocene Izúcar de Matamoros Evaporites (Cuayuca Formation) in Mexico base on the parsimony analisis of endemicity: Palynology, 32, 231-252.

Ramírez-Arriaga, E., Prámparo, M., Guerstein, R., Martínez Hernández, E., GalvánEscobedo, I.G., Corona-Esquivel, R., Marmolejo-Rodríguez, A.J., 2012a, Marine and Continental Assemblages from San Gregorio Formation (Late Oligocene-Early Miocene), La Bocana Region, Baja California
Sur, México (resumen), en Cordilleran Section 108 $8^{\text {th }}$ Annual Meeting, Querétaro, México, 44(3), 15.

Ramírez-Arriaga, E., Prámparo, M., MartínezHernández, E., Nieto-Samaniego, Á., Dávalos-Álvarez, O., Macías-Romo, C., Valiente-Banuet, A., 2012b, Nuevos hallazgos de la flora miocénica en la Formación Tehuacán, México (resumen en versión electrónica), Convención Nacional Geológica 2012, World Trade Center, Ciudad de México, disponible en < http://sociedadgeologica.org.mx/galeria_ convencion_2012.html>, consultado 26 de mayo de 2013.

Ramírez-Arriaga, E., Helenes-Escamilla, J., Prámparo, M., Valiente-Banuet, A., Carreño, A.L., 2013, Late Oligocene-Early Miocene palynoflora from San Gregorio Formation Baja California Sur, México (resumen), en Clark, M., Lucas-Clark, J. y Fisk, L. H. (eds.), AASP- The Palynological Society $46^{\text {th }}$ Annual Meeting, San Francisco, California, U.S.A., 165-166.

Ramírez-Arriaga, E., Prámparo, M., NietoSamaniego, Á., Martínez-Hernández, E., Valiente-Banuet, A., Macías-Romo, C., Dávalos-Álvarez, O., 2014a, Palynological evidence for Middle Miocene vegetation in the Tehuacan Formation of Puebla, Mexico: Palynology, 38(1), 1-27.

Ramírez-Arriaga, E., Reyes-Salas, M., MartínezHernández, E., Ángeles-García, S., 2014b, Polen fósil de Onagraceae Corsinipollenites y Corsinipollis) recuperado de paleocuencas Cenozoicas en Puebla y Baja California Sur, México: Acta Microscópica, 23(2), 111-121.

Rosales-Lomelí, J., Ayala-Nieto, M., MartínezHernández, E., 1992, Investigaciones palinológicas de fósiles terciarios en una columna de la Cuenca Tampico-Misantla, Veracruz, México, Proyecto CAO-1405, 27 p. 
Rzedowski,J., 1996, Análisis preliminar de la flora vascular de los bosques mesófilos de montaña de México: Acta Botánica Mexicana, 35, 25-44.

Rzedowski, J., 2006, Vegetación de México: México, Comisión Nacional para el Conocimiento y Uso de la Biodiversidad, $505 \mathrm{p}$.

Rzedowski, J., Calderón de Rzedowski, G., 1987, El bosque tropical caducifolio de la región mexicana del Bajío: Trace, 12, 12-21.

Sánchez-Rodríguez, E.V., López-Mata, L., García-Moya, E., Cuevas-Guzmán, R., 2003, Estructura, composición florística y diversidad de especies leñosas de un bosque mesófilo de montaña en la sierra de Manantlán, Jalisco: Boletín de la Sociedad Botánica de México, 73, 17-34.

Schwennicke, T., 1994, Deep and shallow water phosphorite bearing strata of the upper Oligocene of Baja California, Mexico (San Juan Member, El Cien Formation): Zentralblatt für Geologie und Palontologie, (Teil I), 567-580.

Schwennicke, T., 1995, Phosphatic grains of the upper Oligocene San Juan member (El Cien Formation) of Baja California Sur, Mexico: Boletín del Departamento de Geología, UniSon, 12(1), 41-64.

Seaby, R.M.H., Henderson, P.A., 2007, Species Diversity and Richness IV: Lymington, Hampshire, Pisces Conservation Ltd., 132 p.

Sluijs, A., Pross, J., Brinkhuis, H., 2005, From greenhouse to icehouse; organic-walled dinoflagellate cysts as paleoenvironmental indicators in the Paleogene: Earth-Science Reviews, 68(3-4), 281-315.

Smith, J.T., 1991, Cenozoic Marine Mollusks and Paleogeography of the Gulf of California, en Dauphin, J.P., Simoneit, B.R.T. (eds.), The Gulf and peninsular province of the
Californias: Oklahoma, U.S.A., American Association of Petroleum Geologists in Tulsa, 637-666.

Srivastava, S.K., 1972, Systematic description of some spores from The Edmonton Formation (Maestrichtian), Alberta, Canada: Palaeontographica, B, 139, 1-46.

Tomasini-Ortíz, A.C., Martínez-Hernández, E., 1984, Palinología del Eoceno-Oligoceno de Simojovel, Chiapas: Paleontología Mexicana, $50,1-60$.

Valiente-Banuet, A., Rumebe, A.V., Verdu, M., Callaway, R.M., 2006, Modern Quaternary plant lineages promote diversity through facilitation of ancient Tertiary lineages: Proceedings of the National Academy of Sciences of the United States of America, 103(45), 16812-16817.

Velázquez-Silva, A.L., 2008, Lluvia de polen en un gradiente altitudinal en la ladera NW del Volcán La Malinche, Tlaxcala México: México, Instituto Politécnico Nacional, tesis de maestría, $64 \mathrm{p}$.

Wiens, J.J., Donoghue, M.J., 2004, Historical biogeography, ecology and species richness: Trends in Ecology \& Evolution, 19(12), 639-644.

Wiens, J.J., Graham, C.H., 2005, Niche Conservatism: Integrating Evolution, Ecology, and Conservation Biology: Annual Review of Ecology, Evolution, and Systematics, 36(1), 519-539.

Zachos, J., Pagani, M., Sloa, L., Thomas, E., Billups, K., 2001, Trends, Rhythms, and Aberrations in Global Climate $65 \mathrm{Ma}$ to present: Science, 292, 686-693.

Zonneveld, K.A.F., Versteegh, G., KodransNsiah, M., 2008, Preservation and organic chemistry of Late Cenozoic organic-walled dinoflagellate cysts: A review: Marine Micropaleontology, 68(1-2), 179-197. 